\title{
KULEUVEN
}

DEPARTMENT OF ECONOMICS

\section{Group consumption with caring individuals}

Laurens CHERCHYE, Sam COSAERT, Thomas DEMUYNCK and Bram DE ROCK

FACULTY OF ECONOMICS AND BUSINESS

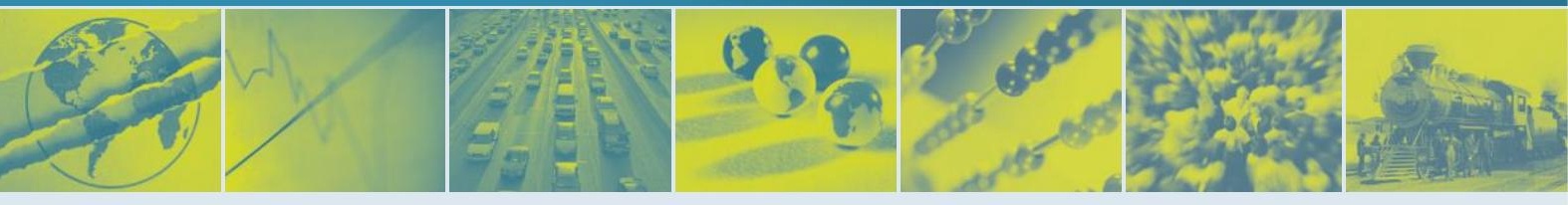

DISCUSSION PAPER SERIES DPS17.15

NOVEMBER 2017 


\title{
Group consumption with caring individuals*
}

\author{
Laurens Cherchye ${ }^{\dagger}$ Sam Cosaert \\ Thomas Demuynck ${ }^{\S}$ and Bram De Rock
}

November 10, 2017

\begin{abstract}
We propose a novel approach to model joint consumption decisions of individuals who care for each other. We assume noncooperative interaction between the different individuals and the within-group consumption outcome critically depends on the degree of caring between the group members. By varying the degree of caring, the model encompasses a whole continuum of group consumption models that are situated between the fully cooperative model (assuming a Pareto optimal outcome) and the noncooperative model without caring (assuming a public good game with voluntary contributions). This feature is used to define a measure for the degree of cooperation within the group, which quantifies how close the observed group behavior is to the fully cooperative benchmark. We also establish a dual characterization of our noncooperative model with caring preferences: we show that the model is dually equivalent to a noncooperative model with non-caring preferences that is characterized by intra-group transfers. Following a revealed preference approach, we derive testable implications of the model for empirical data. Finally, we also use our model to analyze decisions made by dyads of children in an experimental setting. We find considerable heterogeneity in the degree of caring (or cooperation) across dyads, which correlates with assertiveness and the degree of interaction within dyads.
\end{abstract}

JEL Classification: D11, D12, D13, C14.

*This is a substantial revision of an earlier paper that circulated under the the title 'Noncooperative household consumption with caring'. We thank Claude d'Aspremont, Rodolphe Dos Santos Ferreira, Arthur Lewbel, Ian Crawford and participants to workshops and seminars in Aix-en-Provence, Barcolona, Chicago, Louvain-La-Neuve, Oxford, Geneva, Namur and Paris for useful discussion. This research has been funded by the Fund for Scientific Research-Flanders (FWO, Belgium) and the Research Fund of KU Leuven. The usual disclaimer applies.

${ }^{\dagger}$ Department of Economics, University of Leuven. E. Sabbelaan 53, B-8500 Kortrijk, Belgium. E-mail: laurens.cherchye@kuleuven.be. Laurens Cherchye gratefully acknowledges the European Research Council (ERC) for his Consolidator Grant 614221

${ }^{\ddagger}$ Luxembourg Institute of Socio-Economic Research (LISER), Labour market department. Porte des Sciences 11, 4366 Esch-sur-Alzette, Luxembourg. E-mail: Sam.Cosaert@liser.lu.

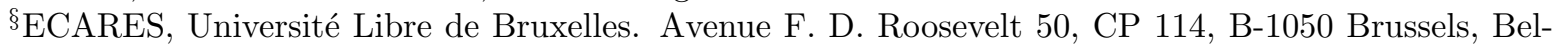
gium. Email: thomas.demuynck@ulb.ac.be.

『ECARES, Université Libre de Bruxelles and University of Leuven. Avenue F. D. Roosevelt 50, CP 114, B-1050 Brussels, Belgium. E-mail: bderock@ulb.ac.be. Bram De Rock gratefully acknowledges FNRS and Belspo. 
Keywords: joint consumption, caring preferences, intra-group cooperation, Nash equilibrium, revealed preferences, children's consumption.

\section{Introduction}

In many instances of group consumption, it is natural to assume that group members care for each other. A prime example is the household consumption context, but there are many other joint decisions (e.g. between family members, friends, ...) for which the caring assumption is an obvious one. In the present paper, we propose a novel consumption model that allows for various degrees of caring between group members. It uses the Beckerian specification of caring preferences (which have also been referred to as altruistic preferences by Becker (1981)). To model the decision process, we assume that there is noncooperative interaction between group members, which means that the intra-group decision process yields intra-group allocations that are Nash equilibria.

Noncooperation versus cooperation. The assumption of noncooperative intra-group interaction has strong theoretical appeal. In particular, any Nash equilibrium is stable in the sense that no group member can increase her/his utility by unilaterally changing her/his strategy. Moreover, using a backward induction argument, one can show that this stability property remains even if we allow for finitely repeated interaction.

The assumption of Nash equilibrium behavior is an obvious one in the context of multiperson group interaction. Particularly, it has been used for modeling the consumption behavior of multi-member households. ${ }^{1}$ A common feature of these existing studies is that they exclude intra-group caring, i.e. the consumption allocation is a Nash equilibrium defined in terms of non-caring preferences. In a consumption setting with both privately and publicly consumed goods, this implies a Nash equilibrium with individuals voluntarily contributing to the public goods. It is well known that, in this case, the resulting level of public goods is generally below the Pareto efficient level.

However, this noncooperative approach also has some deficiencies. First of all, it is frequently unrealistic to assume that group members only care about their own wellbeing. This calls for including caring preferences. Second, group consumption behavior is often likely to overcome free-rider problems associated with public consumption -at least to some extent. Specifically, one may expect that repeated interaction and (nearly) perfect information increase the probability that household members develop welfare enhancing mechanisms to overrule such problems.

At the other extreme, the fully cooperative model assumes that group members reach a Pareto-optimal allocation, i.e. no group member can increase her/his utility without decreasing the utility of any other member. This has become the workhorse model for the empirical analysis of multi-member household behavior. ${ }^{2}$ But the relevance of the

\footnotetext{
${ }^{1}$ See, for example, Leuthold (1968), Bourguignon (1984), Ulph (1988), Kooreman and Kapteyn (1990), Browning (2000), Chen and Woolley (2001), Lechene and Preston (2005, 2011), Browning, Chiappori and Lechene (2010), Cherchye, Demuynck and De Rock (2011), Boone, van der Wiel, van Soest and Vermeulen (2014) and Flinn, Todd and Zhang (2017).

${ }^{2}$ See, for example, Apps and Rees (1988), Chiappori (1988, 1992), Browning and Chiappori (1998), Chiappori and Ekeland (2006, 2009) and Cherchye, De Rock and Vermeulen (2007, 2011). Following
} 
cooperative model has also been advocated in alternative settings of multi-member group consumption (such as committees, clubs, villages and other local organizations). ${ }^{3}$ The premise of efficient behavior can be defended in three ways (see, for example, Browning and Chiappori (1998)). First of all, under perfect information and with repeated interactions Pareto optimal allocations can be stable as long as all members are sufficiently patient. Second, the Pareto outcome is seen as a most natural generalization for multi-member groups of the assumption of utility maximization in a single agent context. Finally, Pareto efficiency is widely used as an assumption in cooperative bargaining models. ${ }^{4}$ In this sense, Pareto optimality is a minimal condition that should be satisfied if the intra-group bargaining process is based on such a cooperative solution concept.

Although we largely agree with these arguments, we also believe that there remains scope for relaxing the efficiency condition. First of all, it is well known that, unless the Pareto optimal allocation exactly coincides with a Nash equilibrium, the cooperative Pareto efficient outcome is not self enforcing. In other words, there will usually be some group member(s) who can increase utility by unilaterally deviating from the Pareto optimal allocation. Second, even if we are in a situation with infinitely repeated interaction, the folk theorem shows that almost every allocation situated between the noncooperative Nash outcome and the Pareto efficient outcome could be stable. In other words, (infinitely) repeated interaction does not necessarily lead to efficient behavior. Finally, the Pareto efficiency assumption has been questioned for the publicly consumed goods. Most notably, it has been argued that the informational requirement and the resulting cost of implementing cooperation may often be unrealistic.

Summarizing, while the fully cooperative model might represent an overly optimistic outlook of group decision processes, we may also argue that the noncooperative model without caring is too pessimistic. Indeed, it appears to us that most group interactions are to be found somewhere between the cooperative and noncooperative benchmarks. In any event, these considerations make it relevant to assess how close the observed consumption behavior is to the fully cooperative benchmark, that is, to evaluate the degree of intra-group cooperation.

Noncooperation with caring. In the following sections, we will present a model of group consumption behavior that encompasses situations between the extreme cases of full cooperation and noncooperation without caring. Formally, our model is equivalent to a noncooperative model where group members have Beckerian caring preferences: each individual optimizes a function that is increasing in the utilities of all group members. ${ }^{5}$ In this set-up, we will derive specific testable restrictions for empirical data. Interestingly, we will also demonstrate that it is possible to empirically recover a measure for the degree

Chiappori $(1988,1992)$, the consumption literature often refers to the cooperative model as the 'collective' model of household behavior.

${ }^{3}$ See, for example, Chiappori and Ekeland (2006).

${ }^{4}$ See Manser and Brown (1980), McElroy and Horney (1981) and Lundberg and Pollak (1993) for applications of bargaining models in a household setting.

${ }^{5}$ In this respect, it is also worth referring to Browning and Lechene (2001), who adopt a similar approach to investigate the relationship between expenditures (on private and public goods) and the intra-group distribution of income. 
of intra-group cooperation which, as we will explain, actually captures caring within the group.

Thus, by introducing caring in the noncooperative framework, our model allows us to combine some attractive properties of the polar cooperative and noncooperative models. At the same time, it solves two main problems associated with these two benchmark models. First of all, as it is based on the concept of a noncooperative Nash equilibrium, it is self enforcing and, hence, stable. Second, by introducing caring between the group members, we depart from the assumption that these members are inherently egoistic (i.e. non-caring). Caring preferences allow for friendship, altruism, love and trust between group members. We believe this assumption to be much more realistic in many group decision situations, where these emotions often do play an important role.

At this point, two remarks are in order. First, it has been demonstrated by Chiappori (1992) that purely egoistic preferences are empirically indistinguishable from caring preferences in the fully cooperative model: the two specifications of individual preferences have exactly the same testable implications under the maintained assumption of Pareto efficient group consumption behavior. In turn, this implies that the caring model (relative to the egoistic model) does not have specific testable implications. ${ }^{6}$ By contrast, as we will argue below, caring preferences do have specific testable implications under the assumption of noncooperative group consumption. As a matter of fact, we will show that it is possible to empirically distinguish a continuum of models characterized by varying degrees of intra-group caring.

Second, d'Aspremont and Dos Santos Ferreira $(2014,2017)$ provide an alternative group consumption model that is situated between the fully cooperative and the noncooperative model. A most important difference with our model is that these authors model 'semicooperative' behavior by parameterizing the trade-off between an individual budget constraint and the group budget constraint (which evaluates the public goods at Lindahl prices). By contrast, the distinguishing feature of our approach is that it combines caring preferences with noncooperative intra-group interaction for modeling the group decision behavior. As motivated above, we believe that accounting for caring preferences is often particularly relevant in the context of joint consumption decisions.

Other contributions. Our consumption model has a number of specific features that are particularly attractive from a theoretical and/or practical perspective. First of all, as indicated above, it allows us to define a measure of intra-group caring that can also be interpreted as quantifying the degree of within-group cooperation. Specifically, we show that it is possible to quantify and estimate the degree of caring within the group; and this gives us an operational measure for the magnitude of intra-group cooperation.

Another interesting feature of our model pertains to its dual representation. Specifically, we will show that the noncooperative model with caring preferences is dually equivalent to a noncooperative model with non-caring preferences that is characterized by intra-group transfers. In fact, the intra-group transfers in the dual model will be directly related to

\footnotetext{
${ }^{6}$ In this respect, Bruyneel, Cherchye, Cosaert, De Rock and Dewitte (2017) show that consumption externalities do have specific testable implications under Pareto efficiency if they are associated with individual commodities consumed by other individuals in the group (rather than with the aggregate wellbeing/utility of the individuals, as in the Beckerian specification of caring preferences).
} 
the above mentioned measure of intra-group cooperation. This duality result parallels the well-known duality between a Pareto optimal allocation and the Lindahl equilibrium, which is often used to provide a decentralized representation for the fully cooperative (Pareto efficient) model of group consumption. As such, we obtain a similar decentralized representation for our newly proposed model.

A further important aspect of our model relates to its empirical applicability. We will show that, although our newly proposed model generalizes the fully cooperative and noncooperative models, it does have useful testable implications for empirical data. To this end, we present a revealed preference characterization of the model in the tradition of Afriat (1967) and Varian (1982): we derive necessary and sufficient conditions for the empirical validity of our model that can be checked by solely using a finite set of observed group consumption bundles and corresponding prices. ${ }^{7}$

Finally, we also demonstrate the practical usefulness of these revealed preference conditions by means of an empirical application, which analyzes decisions made by dyads of children in an experimental setting. We will observe considerable differences in the degree of cooperation across the dyads in our data set. By quantifying this variation by our measure of intra-group cooperation/caring, we can then relate it to observable dyad characteristics. It will appear that particularly the degree of assertiveness and intra-dyad friendship are positively correlated with cooperative behavior, whereas average social skills can not be linked to the degree of intra-group caring.

Outline. The rest of this paper unfolds as follows. Section 2 formally defines our theoretical model of noncooperative group consumption with caring individuals. Section 3 develops its dual (decentralized) representation. Section 4 introduces the testable revealed preference implications of our model. Section 5 presents our empirical application to dyads of children. Section 6 concludes.

\section{A noncooperative model with caring preferences}

We consider a group with two members, $A$ and $B .^{8}$ The group decides over the purchase of a bundle of $N$ private goods, denoted by $q \in \mathbb{R}_{+}^{N}$, and a bundle of $K$ intra-group public goods, denoted by $Q \in \mathbb{R}_{+}^{K}$. We remark that this assumes that each good is either private (in $q$ ) or public (in $Q$ ). Further, it excludes externalities associated with privately consumed quantities. Importantly, however, our setting can actually account for such externalities. Specifically, if an individual is the exclusive consumer of a particular private good, then we can account for externalities for this good by formally treating it as a public good. Throughout, we will treat the first private good as a numeraire and we will assume that the consumption of the numeraire and all public goods is strictly positive in all group

\footnotetext{
${ }^{7}$ See also Samuelson (1938), Houthakker (1950) and Diewert (1973) for seminal contributions to the revealed preference approach to modeling (in case single agent) consumption behavior.

${ }^{8}$ This focus on two-member groups is mainly to keep the exposition simple. Also, our empirical application in Section 5 will consider an experimental setting with two-person groups. At this point, however, it is worth emphasizing that our following analysis can readily be extended to groups with more than two members.
} 
equilibria. $^{9}$

In what follows, we will first formalize our assumptions regarding the preferences and the strategies of the group members. Subsequently, we will formally define and characterize the group equilibrium in terms of our model.

Preferences. Our analysis starts from a set of decision situations $T$. In each situation $t$, the group faces a price vector $p_{t} \in \mathbb{R}_{++}^{N}$ for the private goods, a price vector $P_{t} \in \mathbb{R}_{++}^{K}$ for the public goods, and a group income $Y_{t} \in \mathbb{R}_{++}$. In addition, members $A$ and $B$ are endowed with situation-dependent concave and increasing (Beckerian) caring functions. We denote these functions by $W_{t}^{A}\left(U^{A}, U^{B}\right)$ and $W_{t}^{B}\left(U^{B}, U^{A}\right)$; in this construction, $U^{A}$ and $U^{B}$ stand for 'egoistic' utility functions which (only) depend on the members' own consumption of private goods $\left(q^{A}\right.$ and $\left.q^{B}\right)$ and the total amount of public goods $(Q)$, i.e. $U^{A}=U^{A}\left(q^{A}, Q\right)$ and $U^{B}=U^{B}\left(q^{B}, Q\right)$. Of course, the vectors representing the individual consumption of the private goods should add up to the total group consumption of these goods, i.e. $q^{A}+q^{B}=q$. In contrast to the caring functions $W_{t}^{A}$ and $W_{t}^{B}$, we assume that the utility functions $U^{A}$ and $U^{B}$ are stable (invariant) across all decision situations $t$ in $T$. Indeed, if these functions were also situation-dependent, then our model would have no testable implications. Further, we will assume that utility functions $U^{A}$ and $U^{B}$ are continuous, concave, non-satiated and non-decreasing in their arguments.

An important feature of our model is that the caring functions $W_{t}^{A}$ and $W_{t}^{B}$ are situation-dependent. This is a natural assumption in a consumption framework with interacting individuals. Specifically, it reflects the idea that the degree of caring or altruism between group members might depend on several (situation-dependent) exogenous variables. ${ }^{10}$ These exogenous variables come in two kinds. On the one hand, exogenous variables may influence the decision process within the group. In a household context, examples of such variables are the state of the marriage market, the state of the labor market, the specific divorce laws and the social attitudes to the roles of men and women within the household. On the other hand, exogenous variables may impact on the emotional state of the group members. Examples of such variables are the amounts of love, friendship, compassion and trust within the group. Both kinds of variables may have a strong influence on the shape of the caring functions. Taking the caring functions to be situation-dependent allows the model to adapt to a change in each of these (often unobserved) variables.

In what follows, we will make one additional assumption to facilitate our technical analysis. Specifically, we use a single crossing (SC) property:

Assumption SC: For all decision situations $t, q^{A}, q^{B} \in \mathbb{R}^{N}$ and $Q \in \mathbb{R}_{+}^{K}$, for $\bar{U}^{A}=$

\footnotetext{
${ }^{9}$ We can relax this assumption by using suitable Lagrange multipliers, but this would only increase notational complexity without adding new insights. In fact, our own empirical application in Section 5 will consider data sets with some components of the public goods equal to zero.

${ }^{10}$ Compare with the discussion in Browning, Chiappori and Lechene (2006). These authors consider (situation-dependent) aggregation of preferences in a cooperative framework. In a household consumption context, the situation-specific exogenous variables are analogous to the so-called extra-environmental parameters in the terminology of McElroy and Horney (1981) or distribution factors in the terminology of Browning, Bourguignon, Chiappori and Lechene (1994).
} 
$U^{A}\left(q^{A}, Q\right)$ and $\bar{U}^{B}=U^{B}\left(q^{B}, Q\right)$, we have that either

$$
\left.\frac{\partial W_{t}^{A}}{\partial U^{B}}\right|_{\left(\bar{U}^{A}, \bar{U}^{B}\right)}=0,
$$

or,

$$
-\left.\frac{\frac{\partial W_{t}^{A}}{\partial U^{A}}}{\frac{\partial W_{t}^{A}}{\partial U^{B}}}\right|_{\left(\bar{U}^{A}, \bar{U}^{B}\right)} \leq-\left.\frac{\frac{\partial W_{t}^{B}}{\partial U^{A}}}{\frac{\partial W_{t}^{B}}{\partial U^{B}}}\right|_{\left(\bar{U}^{B}, \bar{U}^{A}\right)} .
$$

The left hand side of the last inequality provides the amount of utility $U^{A}$ that $A$ is willing to subsume to compensate a one unit increase in $U^{B}$. In other words, it gives the slope of the indifference curve of the function $W_{t}^{A}$ in $\mathbb{R}^{2}$ space through the point $\left(\bar{U}^{A}, \bar{U}^{B}\right)$, i.e. the marginal rate of substitution between $U^{A}$ and $U^{B}$. Assumption $\mathrm{SC}$ states that, for every combination of utilities $\bar{U}^{A}$ and $\bar{U}^{B}$, the slope of the indifference curve for $W_{t}^{A}$ through this point is steeper than the slope of the indifference curve of $W_{t}^{B}$ through this point. Intuitively, this single crossing condition implies that, when compared to member $B$, member $A$ gives at least the same weight to her own utility $U^{A}$ as to the utility of the other member $U^{B}$. Symmetrically, $B$ gives relatively more weight to $U^{B}$ than to $U^{A}$ in comparison to $A$. We believe this to be an intuitively plausible assumption. Observe that Assumption SC is entirely ordinal. In other words, it is insensitive to any monotonic transformation of $W_{t}^{A}, W_{t}^{B}, U^{A}$ or $U^{B}$.

Strategies. In order to combine noncooperation and caring in one and the same formal model, we make the following assumption regarding the group members' strategies. At every decision situation $t$, each group member decides on three bundles: member $A$ chooses the private bundles $q_{t}^{A, A}, q_{t}^{A, B} \in \mathbb{R}_{+}^{N}$ and the public bundle $Q_{t}^{A} \in \mathbb{R}_{+}^{K}$; and, similarly, member $B$ chooses $q_{t}^{B, B}, q_{t}^{B, A} \in \mathbb{R}_{+}^{N}$ and $Q_{t}^{B} \in \mathbb{R}_{+}^{K}$. We interpret this as follows: the bundle $q_{t}^{A, A}$ is the bundle of private goods that member $A$ buys for herself, $q_{t}^{A, B}$ is the bundle of private goods that $A$ buys for the other member $B$, and $Q_{t}^{A}$ is the contribution to the bundle of public goods purchased by $A$. The meaning of $q_{t}^{B, B}, q_{t}^{B, A}$ and $Q_{t}^{B}$ is directly analogous. Of course, we must have $q_{t}^{A, A}+q_{t}^{B, A}=q_{t}^{A}, q_{t}^{B, B}+q_{t}^{A, B}=q_{t}^{B}$ and $Q_{t}^{A}+Q_{t}^{B}=Q_{t}$.

It is standard in the literature on noncooperative group behavior to explicitly distinguish between $A$ and $B$ 's contribution to the group's public consumption (for example, Lechene and Preston (2005, 2011), and d'Aspremont and Dos Santos Ferreira (2014) make similar distinctions). However, the fact that we allow $A$ and $B$ to buy private goods for each other may seem a bit unconventional. In most models (of noncooperative behavior) it is assumed that members only buy private goods for themselves, i.e. $A$ chooses $q_{t}^{A}$ and $B$ chooses $q_{t}^{B}$. Our distinction between $q_{t}^{M, M}$ and $q_{t}^{M, L}$ (for $M, L \in\{A, B\}, M \neq L$ ) directly relates to the specificity of our model, i.e. it accounts for caring preferences in a noncooperative setting.

Let us explain this last point in some more detail. In a noncooperative model without caring preferences, it seems intuitive that individual members will not buy private goods for the other. By contrast, in the case of intra-group caring, one group member may well 
benefit from increasing the private consumption of the other member. Our distinction between $q_{t}^{M, M}$ and $q_{t}^{M, L}$ exactly takes this into account. ${ }^{11}$

Equilibrium. We will first introduce our new concept of group equilibrium in general terms. Subsequently, we will show that the concept encompasses the fully cooperative equilibrium and the noncooperative equilibrium without caring as limiting cases. This demonstrates the generality of our model. Furthermore, it will enable us to interpret our measure of intra-group caring as quantifying the degree of within-group cooperation, i.e. the measure allows us to distinguish between different consumption models characterized by different of degrees of cooperation.

We assume that in equilibrium both members maximize their caring functions given the decisions of the other members, i.e. we assume a noncooperative Nash equilibrium. More formally, at decision situation $t$, member $A$ solves the following optimization problem (OP-A):

$$
\begin{aligned}
&\left(q_{t}^{A, A}, q_{t}^{A, B}, Q_{t}^{A}\right)=\underset{\left(q^{A, A}, q^{A, B}, Q^{A}\right)}{\arg \max } W_{t}^{A}\left(U^{A}\left(q^{A}, Q\right), U^{B}\left(q^{B}, Q\right)\right) \\
& \text { s.t. } p_{t}^{\prime}\left(q^{A}+q^{B}\right)+P_{t}^{\prime} Q \leq Y_{t}, \\
& q^{A, A}+q_{t}^{B, A}=q^{A}, \\
& q^{A, B}+q_{t}^{B, B}=q^{B}, \\
& Q^{A}+Q_{t}^{B}=Q .
\end{aligned}
$$

Similarly, $B$ solves (OP-B):

$$
\begin{aligned}
&\left(q_{t}^{B, B}, q_{t}^{B, A}, Q_{t}^{B}\right)=\underset{\left(q^{B, B}, q^{B, A}, Q^{B}\right)}{\arg \max } W_{t}^{B}\left(U^{B}\left(q^{B}, Q\right), U^{A}\left(q^{A}, Q\right)\right) \\
& \text { s.t. } p_{t}^{\prime}\left(q^{A}+q^{B}\right)+P_{t}^{\prime} Q \leq Y_{t} \\
& q_{t}^{A, A}+q^{B, A}=q^{A} \\
& q_{t}^{A, B}+q^{B, B}=q^{B} \\
& Q_{t}^{A}+Q^{B}=Q .
\end{aligned}
$$

An allocation that solves both problems simultaneously is called a group equilibrium with caring.

Definition 1 An allocation $\left\{q_{t}^{A, A}, q_{t}^{A, B}, q_{t}^{B, B}, q_{t}^{B, A}, Q_{t}^{A}, Q_{t}^{B}\right\}$ is a group equilibrium with caring if and only if it simultaneously solves $\boldsymbol{O P}-\boldsymbol{A}$ and $\boldsymbol{O P}-\boldsymbol{B}$.

Our new model enables us to define a measure of intra-group caring. To formalize this idea, let $\partial U^{M}\left(q^{M}, Q\right) / \partial q_{1}^{M}$ represent the marginal utility of the numeraire (i.e. the first

\footnotetext{
${ }^{11}$ Browning, Chiappori and Lechene (2010) suggest a similar idea in the context of a noncooperative model with one private good and one public good, where one individual has caring preferences while the other individual is egoistic. In fact, a similar mechanism also underlies Becker's rotten kid theorem.
} 
private good) for member $M \in\{A, B\}$ at the allocation $\left\{q^{M}, Q\right\}$. Then, for a public good $k$ we define ${ }^{12}$

$$
\left.\tau_{k}^{M}\left(q^{M}, Q\right) \equiv \frac{\frac{\partial U^{M}}{\partial Q_{k}}}{\frac{\partial U^{M}}{\partial q_{1}^{M}}}\right|_{\left(q^{M}, Q\right)} .
$$

In words, the function value $\tau_{k}^{M}\left(q^{M}, Q\right)$ gives member $M$ 's marginal willingness to pay (MWTP) for an additional unit of $k$ at $\left\{q^{M}, Q\right\}$. We can now derive the following result. (The proofs of our main results are given in Appendix A.)

Proposition 1 Let $\left\{q_{t}^{A, A}, q_{t}^{A, B}, q_{t}^{B, B}, q_{t}^{B, A}, Q_{t}^{A}, Q_{t}^{B}\right\}$ be a group equilibrium with caring. Then, there exist numbers $\theta_{t}^{A}, \theta_{t}^{B} \in[0,1]$ such that for all public goods $k$ :

$$
\max \left\{\begin{array}{c}
\tau_{k}^{A}\left(q_{t}^{A}, Q_{t}\right)+\theta_{t}^{B} \tau_{k}^{B}\left(q_{t}^{B}, Q_{t}\right) ; \\
\tau_{k}^{B}\left(q_{t}^{B}, Q_{t}\right)+\theta_{t}^{A} \tau_{k}^{A}\left(q_{t}^{A}, Q_{t}\right)
\end{array}\right\}=P_{t, k} .
$$

It follows from the proof of this proposition that the values of the indices $\theta_{t}^{A}$ and $\theta_{t}^{B}$ are determined by the curvatures of the caring functions $W_{t}^{A}$ and $W_{t}^{B}$ at equilibrium, which actually capture the degree of intra-group caring. ${ }^{13}$ Assumption SC guarantees that $\theta_{t}^{A}$ and $\theta_{t}^{B}$ are both contained in the unit interval. In the next section, we will use the dual representation of our consumption model to provide a specific equilibrium interpretation for the equality condition in Proposition 1.

To further enhance the intuition of our newly proposed model, we consider the two natural benchmark cases, i.e. the fully cooperative model and the noncooperative model without caring. In terms of Definition 1 (and problems OP-A and OP-B), if the caring functions $W_{t}^{A}$ and $W_{t}^{B}$ coincide (i.e. $W_{t}^{A}=W_{t}^{B}=W_{t}$ ), then both members optimize the same objective function. By construction, this implies a cooperative equilibrium (i.e. a Pareto optimal intra-group allocation). In this case, the caring function $W_{t}$ corresponds to a so-called generalized (Samuelson) group welfare function (see, for example, Apps and Rees (2009)). By varying $W_{t}$, any Pareto efficient allocation can be reached as a group equilibrium with caring. By contrast, if the caring functions reduce to 'egoistic' functions (i.e. $W_{t}^{A}\left(U^{A}, U^{B}\right)=U^{A}$ and $\left.W_{t}^{B}\left(U^{B}, U^{A}\right)=U^{B}\right)$, then the group equilibrium

${ }^{12}$ Throughout, we use $\frac{\partial U^{M}}{\partial q_{n}^{M}}$ for the partial derivative of the utility function $U^{M}$ with respect to the consumption quantity of the private good $n$, and $\frac{\partial U^{M}}{\partial Q_{k}}$ for the partial derivative of the function $U^{M}$ associated with the quantity of the public good $k$.

$$
{ }^{13} \text { Formally, we have } \theta_{t}^{A}=\left(\frac{\partial W_{t}^{B}}{\partial U^{A}} / \frac{\partial W_{t}^{B}}{\partial U^{B}}\right)\left(\frac{\partial U^{A}}{\partial q_{1}^{A}} / \frac{\partial U^{B}}{\partial q_{1}^{B}}\right) \text { and } \theta_{t}^{B}=
$$
$\left(\frac{\partial W_{t}^{A}}{\partial U^{B}} / \frac{\partial W_{t}^{A}}{\partial U^{A}}\right)\left(\frac{\partial U^{B}}{\partial q_{1}^{B}} / \frac{\partial U^{A}}{\partial q_{1}^{A}}\right)$, where all partial derivatives are evaluated at the allocation $\left\{q_{t}^{A}, q_{t}^{B}, Q_{t}\right\}$. In words, $\theta_{t}^{A}$ equals the ratio of member $B$ 's marginal valuation for a unit increase of the numeraire quantity for member $A$ (which enters the caring function $W_{t}^{B}$ through $U^{A}$ ) relative to his marginal valuation for the same increase of the numeraire quantity for his own (which enters $W_{t}^{B}$ through $U^{B}$ ). Likewise, the variable $\theta_{t}^{B}$ equals the ratio of $A$ 's marginal valuation for a unit increase of the numeraire quantity for $B$ relative to her marginal valuation for the same quantity increase for her own. 
reduces to a noncooperative equilibrium without caring (i.e. a standard public good game with voluntary contributions). Our model is general in that it also captures all possible equilibrium situations between the fully cooperative equilibrium and the noncooperative equilibrium without caring.

Using the same two benchmark models, we can effectively interpret the indices $\theta_{t}^{A}$ and $\theta_{t}^{B}$ in Proposition 1 as capturing the degree of cooperation at the equilibrium intra-group allocation. First, in a cooperative equilibrium the MWTP functions $\tau_{k}^{M}$ coincide with the so-called Lindahl prices. In particular, it is well known that any Pareto efficient allocation $\left\{q_{t}^{A}, q_{t}^{B}, Q_{t}\right\}$ must satisfy the Lindahl-Bowen-Samuelson conditions (see, for example, Samuelson (1954)). And, thus, we get for each public good $k$ :

$$
\tau_{k}^{A}\left(q_{t}^{A}, Q_{t}\right)+\tau_{k}^{B}\left(q_{t}^{B}, Q_{t}\right)=P_{t, k}
$$

In words, the sum of the members' MWTP must sum to the market prices. This case coincides with $\theta_{t}^{A}=\theta_{t}^{B}=1$ in Proposition 1 .

We next turn to the noncooperative model. In this case we get the following equilibrium condition for every public good $k$ :

$$
\max \left\{\begin{array}{c}
\tau_{k}^{A}\left(q_{t}^{A}, Q_{t}\right) \\
\tau_{k}^{B}\left(q_{t}^{B}, Q_{t}\right)
\end{array}\right\}=P_{t, k}
$$

see, for example, Cherchye, Demuynck and De Rock (2011). Thus, this case corresponds to $\theta_{t}^{A}=\theta_{t}^{B}=0$ in Proposition 1 .

More generally, if the indices $\theta_{t}^{A}$ and $\theta_{t}^{B}$ are closer to unity, the group will behave more as in the cooperative model. The duality result in Section 4 will provide an additional interpretation of $\theta_{t}^{A}$ and $\theta_{t}^{B}$ as quantifying the degree of intra-group cooperation of each member. In Section 5 we will show that it is possible to empirically recover the values of $\theta_{t}^{A}$ and $\theta_{t}^{B}$. In this respect, we also note that $\max \left\{\theta_{t}^{A}, \theta_{t}^{B}\right\}<1$ implies (because of Proposition 1)

$$
\tau_{k}^{A}\left(q_{t}^{A}, Q_{t}\right)+\tau_{k}^{B}\left(q_{t}^{B}, Q_{t}\right)>P_{t, k},
$$

which reveals Pareto inefficient behavior. As such, $\theta_{t}^{A}$ and $\theta_{t}^{B}$ also indicate the extent of Pareto (in)efficiency at each decision situation $t$.

As a final remark, we note that the values of $\theta_{t}^{A}$ and $\theta_{t}^{B}$ are situation-dependent in the general version of our model. In practice, one may impose $\theta_{t}^{A}=\theta^{A}$ and $\theta_{t}^{B}=\theta^{B}$ for all $t$, which thus assumes a constant degree of intra-group cooperation over all decision situations. Again, this encompasses the fully cooperative model (with $\theta^{A}=\theta^{B}=1$ ) and the noncooperative model without caring (with $\theta^{A}=\theta^{B}=0$ ) as limiting cases. As a specific illustration, we will consider such constant intra-group cooperation in our empirical application in Section 5 .

\section{A duality result}

The second fundamental theorem of welfare economics provides one of the most important theoretical insights related to the concept of Pareto efficiency. Specifically, provided that some regularity conditions are satisfied, any Pareto optimal allocation can be dually 
characterized in terms of a suitable income distribution and by making use of individual Lindahl prices for the publicly consumed goods (see, for example, Bergstrom (1976)). This dual characterization of Pareto optimality has often been used to provide a decentralized two-stage representation of the fully cooperative model of group consumption: in the first stage, the group divides the total income over the group members; in the second stage, each individual member chooses a consumption allocation that maximizes her/his utility subject to the personalized budget constraint defined in the first stage.

In this section, we will develop a similar duality result for the noncooperative model with caring preferences that we introduced above: we will show that this model is dually equivalent to a noncooperative model with non-caring preferences that is characterized by intra-group transfers. The magnitude of these transfers will be directly related to the MWTP functions $\tau_{k}^{A}$ and $\tau_{k}^{B}$ and the indices $\theta_{t}^{A}$ and $\theta_{t}^{B}$ introduced in the previous section. In turn, this duality result implies a decentralized representation of the model that contains two stages. As we will explain, this representation will provide a further motivation to interpret $\theta_{t}^{A}$ and $\theta_{t}^{B}$ as measuring the degree of intra-group cooperation.

Before formally stating the duality result, we first explain the two stages of the noncooperative group model with transfers. In the first stage, the total group income $Y_{t}$ is divided between $A$ and $B$, which defines the individual incomes $Y_{t}^{A}$ and $Y_{t}^{B}$ (with $Y_{t}^{A}+Y_{t}^{B}=Y_{t}$ ). Here, we abstract from explicitly modeling this first step. Similar to our treatment of caring functions in the previous section, this intra-group income distribution can be seen as a function of situation-dependent exogenous variables. The idea of an intra-group income distribution resembles the so-called 'sharing rule' concept that applies to the fully cooperative model: in the decentralized representation of this model, the sharing equally defines the within-group income distribution underlying the (in casu Pareto efficient) group consumption decisions. ${ }^{14}$

In the second stage of the allocation process, each group member $M(=A$ or $B)$ decides on the optimal level of her/his own private consumption and the own contribution to the level of public goods, by maximizing her/his own utility $U^{M}\left(q^{M}, Q\right)$ subject to a personalized budget constraint defined by the individual income. In doing so, the individual faces the price vectors $p_{t}$ and $P_{t}$ for her/his choice of private consumption $q_{t}^{M}$ and public contribution $Q_{t}^{M}$. In addition, each individual receives a transfer from the other individual per unit of public good that she/he purchases. We denote these transfers for each public good $k$ by $\sigma_{t, k}^{A}$ and $\sigma_{t, k}^{B} ; \sigma_{t}^{A}$ and $\sigma_{t}^{B}$ represent the corresponding vectors of intra-group transfers.

There are at least two interpretations for these intra-group transfers related to public goods. First, one can see these transfers as voluntary contributions: as $B$ benefits from the purchase of $Q_{t, k}^{A}$, it may be the case that she/he is willing to contribute to the purchase

\footnotetext{
${ }^{14}$ In fact, Chiappori $(1988,1992)$ originally introduced this sharing rule concept for the model without public goods. In the literature on the cooperative model, a refinement of the concept that accounts for public goods is the so-called 'conditional' sharing rule. This concept captures how the group shares the income to be spent on private consumption for the given level of public consumption; see, for example, Blundell, Chiappori and Meghir (2005) for discussion. As such, this first step income distribution concept is not fully comparable to ours, which is not conditional on the level of public consumption. See also Cherchye, De Rock, Lewbel and Vermeulen (2015) for a more general sharing rule concept that accounts for public consumption under cooperative behavior, which is close in spirit to the sharing idea associated with the dual representation of our caring model.
} 
of this bundle. Next, one can also interpret them as representing an implicit tax that $B$ has to pay for the benefit of receiving $Q_{t, k}^{A}$. Both interpretations express that intra-group transfers (i.e. a given specification of $\sigma_{t}^{A}$ and $\sigma_{t}^{B}$ ) refer to the degree of (voluntary or obligatory) cooperation within the group.

Summarizing, at each decision situation $t$, member $A$ faces the following dual optimization problem (DOP-A):

$$
\begin{aligned}
\left\{q_{t}^{A}, Q_{t}^{A}\right\} \in \arg \max _{q^{A}, Q^{A}} U^{A}\left(q^{A}, Q^{A}+Q_{t}^{B}\right), \\
\text { s.t. } p_{t}^{\prime} q^{A}+\left(P_{t}-\sigma_{t}^{B}\right)^{\prime} Q^{A}+\sigma_{t}^{A \prime} Q_{t}^{B} \leq Y_{t}^{A} .
\end{aligned}
$$

Similarly, B solves (DOP-B):

$$
\begin{aligned}
\left\{q_{t}^{B}, Q_{t}^{B}\right\} & \in \underset{q^{B}, Q^{B}}{\arg \max } U^{B}\left(q^{B}, Q^{B}+Q_{t}^{A}\right), \\
\text { s.t. } & p_{t}^{\prime} q^{B}+\left(P_{t}-\sigma_{t}^{A}\right)^{\prime} Q^{B}+\sigma_{t}^{B \prime} Q_{t}^{A} \leq Y_{t}^{B} .
\end{aligned}
$$

It is easy to see that the two budget constraints add up to the group budget constraint at equilibrium (i.e. $p_{t}^{\prime} q_{t}+P_{t}^{\prime} Q_{t} \leq Y_{t}$ ).

Importantly, the noncooperative model under study does not explicitly consider caring preferences: in contrast to the model discussed in the previous section, the problems DOPA and DOP-B do not include the caring functions $W_{t}^{A}$ and $W_{t}^{B}$ but only use the 'egoistic' functions $U^{A}$ and $U^{B}$. However, as we will explain, our following concept of a group equilibrium with transfers accounts for caring preferences in an indirect way.

Definition 2 An allocation $\left\{q_{t}^{A}, q_{t}^{B}, Q_{t}^{A}, Q_{t}^{B}\right\}$ is a group equilibrium with transfers if and only if it simultaneously solves $\boldsymbol{D O P}-\boldsymbol{A}$ and $\boldsymbol{D O P}-\boldsymbol{B}$ and, in addition, there exist $\theta_{t}^{A}$ and $\theta_{t}^{B}$ such that for all public goods $k$ :

$$
\sigma_{t, k}^{A}=\theta_{t}^{A} \tau_{k}^{A}\left(q_{t}^{A}, Q_{t}\right) \quad \text { and } \quad \sigma_{t, k}^{B}=\theta_{t}^{B} \tau_{k}^{B}\left(q_{t}^{B}, Q_{t}\right) .
$$

In this definition, an equilibrium allocation requires that each member $M$ 's intra-group transfer related to public good $k\left(\sigma_{t, k}^{M}\right)$ is proportional to $M$ 's MWTP for $k\left(\tau_{k}^{M}\left(q_{t}^{M}, Q_{t}\right)\right)$. The factor of proportionality is giving by the index $\theta_{t}^{M}$. Definition 2 establishes a direct link between the noncooperative model with caring introduced in the previous section (with problems OP-A and OP-B) and the two-stage allocation process discussed here (with problems DOP-A and DOP-B). In the previous section, we argued that the curvatures of the caring functions $W_{t}^{A}$ and $W_{t}^{B}$ define $\theta_{t}^{A}$ and $\theta_{t}^{B}$. As such, the condition on the intra-group transfers in Definition 2 indirectly incorporates caring preferences in the group equilibrium under consideration.

Interestingly, Definition 2 provides an additional interpretation of each index $\theta_{t}^{M}$ in terms of intra-group cooperation. Given member M's MWTP for the public good $k$ $\left(\tau_{k}^{M}\left(q_{t}^{M}, Q_{t}\right)\right), \theta_{t}^{M}$ captures the transfer $M$ is willing to give to the other member $L(L \neq M)$ if $L$ purchases an additional unit of good $k$. In the fully cooperative case, $M$ is willing to donate the full amount $\tau_{k}^{M}\left(q_{t}^{B}, Q_{t}\right)$ to $L$, which means $\theta_{t}^{M}=1$. In this case, Definition 2 coincides with the standard definition of a Lindahl equilibrium. By contrast, in the noncooperative case without caring, $M$ will not donate anything to $L$, so that $\theta_{t}^{M}=0$. Now, 
Definition 2 reduces to the usual definition of a noncooperative equilibrium without caring. Apart from these fully cooperative and noncooperative cases, Definition 2 also includes the intermediate case in which $M$ picks a number $\theta_{t}^{M}$ between 0 and 1 such that she/he donates a fraction $\theta_{t}^{M}$ of $\tau_{k}^{M}\left(q_{t}^{M}, Q_{t}\right)$ to $L$. Generally, a higher (lower) $\theta_{t}^{M}$ means that $M$ is willing to cooperate more (less) with $L$.

Using Definition 2, we get the following first order conditions for DOP-A and DOP-B with respect to the public good $k$ :

$$
\max \left\{\begin{array}{l}
\tau_{k}^{A}\left(q_{t}^{A}, Q_{t}\right)+\theta_{t}^{B} \tau_{k}^{B}\left(q_{t}^{B}, Q_{t}\right) ; \\
\tau_{k}^{B}\left(q_{t}^{B}, Q_{t}\right)+\theta_{t}^{A} \tau_{k}^{A}\left(q_{t}^{A}, Q_{t}\right)
\end{array}\right\}=P_{t, k} .
$$

This condition is identical to the equilibrium condition in Proposition 1. However, the underlying interpretation is different, because we now start from the optimization problems DOP-A and DOP-B rather than OP-A and OP-B.

By considering $\theta_{t}^{A}$ and $\theta_{t}^{B}$ as capturing intra-group transfers, we can provide an intuitive equilibrium interpretation to the above equality condition. To see this, let us consider the two possible inequality situations. First, if

$$
\tau_{k}^{A}\left(q_{t}^{A}, Q_{t}\right)+\theta_{t}^{B} \tau_{k}^{B}\left(q_{t}^{B}, Q_{t}\right)>P_{t, k}
$$

then the total amount that $A$ is willing to spend for an additional unit of public good $k$ (i.e. $A$ 's MWTP plus the fraction $\theta_{t}^{B}$ of $B$ 's MWTP) exceeds the price $A$ has to pay (i.e. $\left.P_{t, k}\right)$. In this case, $A$ will effectively increase her holdings of good $k$. A directly analogous interpretation applies to the situation,

$$
\tau_{k}^{B}\left(q_{t}^{B}, Q_{t}\right)+\theta_{t}^{A} \tau_{k}^{A}\left(q_{t}^{A}, Q_{t}\right)>P_{t, k} .
$$

And, thus,

$$
\max \left\{\begin{array}{l}
\tau_{t}^{A}\left(q_{t}^{A}, Q_{t}\right)+\theta_{t}^{B} \tau_{t}^{B}\left(q_{t}^{B}, Q_{t}\right) ; \\
\tau_{t}^{B}\left(q_{t}^{B}, Q_{t}\right)+\theta_{t}^{A} \tau_{k}^{A}\left(q_{t}^{A}, Q_{t}\right)
\end{array}\right\}>P_{t, k},
$$

implies a disequilibrium. Similarly, if we have

$$
\max \left\{\begin{array}{l}
\tau_{k}^{A}\left(q_{t}^{A}, Q_{t}\right)+\theta_{t}^{B} \tau_{k}^{B}\left(q_{t}^{B}, Q_{t}\right) ; \\
\tau_{k}^{B}\left(q_{t}^{B}, Q_{t}\right)+\theta_{t}^{A} \tau_{k}^{A}\left(q_{t}^{A}, Q_{t}\right)
\end{array}\right\}<P_{t, k},
$$

then either $A$ or $B$ (whoever contributes positively to good $k$ ) will want to decrease her/his contribution to $k$. Again, this implies a disequilibrium situation.

We are now in a position to establish the dual equivalence result mentioned above. Specifically, the following proposition implies that the model with caring and the model with transfers are empirically indistinguishable.

Proposition 2 Let $U^{A}$ and $U^{B}$ be a pair of utility functions. Then, the following holds for any decision situation $t$ :

1. Suppose $\left\{q_{t}^{A, A}, q_{t}^{A, B}, q_{t}^{B, B}, q_{t}^{B, A}, Q_{t}^{A}, Q_{t}^{B}\right\}$ is a group equilibrium with caring.

Then, there exist individual incomes $Y_{t}^{A}$ and $Y_{t}^{B}$ (with $Y_{t}^{A}+Y_{t}^{B}=Y_{t}$ ) and indices $\theta_{t}^{A}$ and $\theta_{t}^{B}$ such that $\left\{q_{t}^{A}, q_{t}^{B}, Q_{t}^{A}, Q_{t}^{B}\right\}$ is a group equilibrium with transfers. 
2. Suppose $\left\{q_{t}^{A}, q_{t}^{B}, Q_{t}^{A}, Q_{t}^{B}\right\}$ is a group equilibrium with transfers.

Then, there exist caring functions $W_{t}^{A}$ and $W_{t}^{B}$ and bundles $q_{t}^{A, A}, q_{t}^{A, B}, q_{t}^{B, B}, q_{t}^{B, A}, Q_{t}^{A}, Q_{t}^{B}$ $\left(\right.$ with $q_{t}^{A}=q_{t}^{A, A}+q_{t}^{A, B}, q_{t}^{B}=q_{t}^{B, A}+q_{t}^{B, B}$ and $\left.Q_{t}=Q_{t}^{A}+Q_{t}^{B}\right)$ such that $\left\{q_{t}^{A, A}, q_{t}^{A, B}\right.$, $\left.q_{t}^{B, B}, q_{t}^{B, A}, Q_{t}^{A}, Q_{t}^{B}\right\}$ is a group equilibrium with caring.

\section{Testable implications}

So far we have focused on the theoretical properties of our model with caring (or, equivalently, with transfers). In this section, we show that the model has useful testable implications for empirical data. Specifically, we will focus on testable conditions in terms of revealed preferences. This revealed preference approach has been successfully applied for empirical analysis of group consumption models: Cherchye, De Rock and Vermeulen (2007, 2011) focus on the cooperative model and Cherchye, Demuynck and De Rock (2011) on the noncooperative model without caring. In addition, recent methodological advances of Blundell, Browning and Crawford $(2003,2008)$ and Cherchye, De Rock, Lewbel and Vermeulen (2015) greatly enhanced the empirical usefulness of this revealed preference approach.

In the consumption literature, empirical studies usually build on a differential characterization (rather than a revealed preference characterization) of consumption models. The specific feature of this differential approach is that it focuses on properties of functions representing consumption behavior (e.g. cost, indirect utility and demand functions), whereas the revealed preference approach (only) uses a finite set of group consumption observations. ${ }^{15}$ In this respect, Cherchye, Demuynck and De Rock (2011) point out that the revealed preference approach has some attractive features as compared to the more common differential approach for analyzing group consumption behavior. Most notably, contrary to existing results for the differential approach, the revealed preference characterization of the noncooperative model (without caring) is independent from (or non-nested with) the characterization of the cooperative model: a set of observations that satisfies the cooperative conditions does not necessarily satisfy the noncooperative conditions, and vice versa. More generally, this implies that models characterized by different degrees of intragroup cooperation (or caring) are independent of each other in terms of their revealed preference characterization. Clearly, this independence makes it interesting to compare the empirical validity of the different models. This is particularly relevant in the present context, as our empirical application in the next section will carry out such a comparison.

Revealed preference characterization. We start from a finite set $T$ of observed decision situations (or 'observations'), i.e. $S=\left\{p_{t}, P_{t}, q_{t}, Q_{t}\right\}_{t \in T}$. We remark that this implies

\footnotetext{
${ }^{15}$ The term 'differential' refers to the fact that the characterization is obtained by integrating and/or differentiating the functional specifications of the fundamentals of the model (e.g. individual preferences). For differential characterizations of group consumption models, see Browning and Chiappori (1998) and Chiappori and Ekeland (2006, 2009), who focused on the cooperative model, Lechene and Preston (2005, 2011), who considered the noncooperative model without caring, and d'Aspremont and Dos Santos Ferreira (2014), who proposed a semicooperative model characterized by trade-offs between individual-level and group-level budget constraints.
} 
minimal conditions on what is observed. In particular, we assume that at each observation $t$ we only observe the price vectors $p_{t}$ and $P_{t}$ and the aggregate consumption bundles $q_{t}$ and $Q_{t}$. Given our discussion in the previous sections, we consider the following definition of rationalizability.

Definition 3 Consider a data set $S=\left\{p_{t}, P_{t}, q_{t}, Q_{t}\right\}_{t \in T}$. We say that $S$ is rationalizable with caring if there exist utility functions $U^{A}$ and $U^{B}$ and, for each decision situation $t$, there exist caring functions $W_{t}^{A}$ and $W_{t}^{B}$ and bundles $q_{t}^{A, A}, q_{t}^{A, B}, q_{t}^{B, B}, q_{t}^{B, A}, Q_{t}^{A}, Q_{t}^{B}$ (with $q_{t}^{A}=q_{t}^{A, A}+q_{t}^{A, B}, q_{t}^{B}=q_{t}^{B, A}+q_{t}^{B, B}$ and $\left.Q_{t}=Q_{t}^{A}+Q_{t}^{B}\right)$ such that $\left\{q_{t}^{A, A}, q_{t}^{A, B}, q_{t}^{B, B}, q_{t}^{B, A}, Q_{t}^{A}, Q_{t}^{B}\right\}$ is a group equilibrium with caring.

Before providing testable revealed preference conditions for rationalizability, we briefly recapture a result of Varian (1982; based on Afriat, 1967). Consider a finite set of $|L|$ observations, i.e. a set $Z=\left\{w_{l}, x_{l}\right\}_{l \in L}$ containing price vectors $w_{l}$ and quantity vectors $x_{l}$. Then, we say that this set $Z$ can be rationalized by a utility function $U$ if each quantity bundle $x_{l}$ maximizes the function $U$ in the following sense:

$$
x_{l} \in \arg \max _{x} U(x) \text { s.t. } w_{l}^{\prime} x \leq w_{l}^{\prime} x_{l} .
$$

Varian (1982) has shown that such a rationalizing utility function $U$ exists if and only if the set $Z$ satisfies the Generalized Axiom of Revealed Preference (GARP).

Definition 4 Consider a set $Z=\left\{w_{l}, x_{l}\right\}_{l \in L}$. For any $l_{1}, l_{2} \in L$, define $x_{l_{1}} R^{D} x_{l_{2}}$ if

$$
w_{l_{1}}^{\prime} x_{l_{1}} \geq w_{l_{1}}^{\prime} x_{l_{2}} .
$$

Next, $x_{l_{1}} R x_{l_{2}}$ if there exists a sequence $r, \ldots, t$ (with $r, \ldots, t \in L$ ) such that

$$
x_{l_{1}} R^{D} x_{r}, \ldots, x_{t} R^{D} x_{l_{2}} .
$$

The set $Z$ satisfies $G A R P$ if, for all $l_{1}, l_{2} \in L, x_{l_{1}} R x_{l_{2}}$ implies

$$
w_{l_{2}}^{\prime} x_{l_{1}} \geq w_{l_{2}}^{\prime} x_{l_{2}} \text {. }
$$

Using Definition 4, we can characterize a data set $S$ that is rationalizable with caring.

Proposition 3 Consider a data set $S=\left\{p_{t}, P_{t}, q_{t}, Q_{t}\right\}_{t \in T}$. The following conditions are equivalent:

1. The data set $S=\left\{p_{t}, P_{t}, q_{t}, Q_{t}\right\}_{t \in T}$ is rationalizable with caring.

2. For all decision situations $t$ and public goods $k$ there exist indices $\theta_{t}^{A}, \theta_{t}^{B} \in[0,1]$, vectors $\tau_{t}^{A}=\left(\tau_{t, 1}^{A}, \ldots, \tau_{t, K}^{A}\right), \tau_{t}^{B}=\left(\tau_{t, 1}^{B}, \ldots, \tau_{t, K}^{B}\right) \in \mathbb{R}_{+}^{K}$, and bundles $q_{t}^{A}, q_{t}^{B} \in \mathbb{R}_{+}^{N}$ such that

$$
\begin{aligned}
& q_{t}^{A}+q_{t}^{B}=q_{t}, \\
& \max \left\{\begin{array}{c}
\tau_{t, k}^{A}+\theta_{t}^{B} \tau_{t, k}^{B} ; \\
\tau_{t, k}^{B}+\theta_{t}^{A} \tau_{t, k}^{A}
\end{array}\right\}=P_{t, k}, \text { and } \\
& \left\{p_{t}, \tau_{t}^{A}, q_{t}^{A}, Q_{t}\right\}_{t \in T} \text { and }\left\{p_{t}, \tau_{t}^{B}, q_{t}^{B}, Q_{t}\right\}_{t \in T} \text { satisfy GARP. }
\end{aligned}
$$


Moreover, it follows that there exists $Q_{t}^{A}, Q_{t}^{B} \in \mathbb{R}_{+}^{K}$ such that

$$
\begin{aligned}
& \text { if } \tau_{t, k}^{A}+\theta_{t}^{B} \tau_{t, k}^{B}<P_{t, k} \text { then } Q_{t, k}^{A}=0 \text { and } Q_{t, k}^{B}=Q_{t, k} \text {, and } \\
& \text { if } \theta_{t}^{A} \tau_{t, k}^{A}+\tau_{t, k}^{B}<P_{t, k} \text { then } Q_{t, k}^{B}=0 \text { and } Q_{t, k}^{A}=Q_{t, k} .
\end{aligned}
$$

The explanation is as follows. The restriction S.1 requires the individual consumption bundles for the private goods to sum to the demanded group bundle of private goods. The restriction S.2 corresponds to the equilibrium condition for the public goods $k$ in Proposition 1 (for a positive consumption of the public good $k$ ). Condition S.3 states that rationalizability implies a GARP condition at the level of individuals $A$ and $B$, which corresponds to the existence of the individual utility functions $U^{A}$ and $U^{B}$ in Definition 3. The specificity of our model is that these GARP conditions use MWTP vectors (i.e. $\tau_{t}^{A}$ and $\tau_{t}^{B}$ ) for evaluating the publicly consumed quantities (i.e. $Q_{t}$ ). Finally, the conditions S.4 and S.5 follow from the fact that, if $\tau_{t, k}^{A}+\theta_{t}^{B} \tau_{t, k}^{B}<P_{t, k}\left(\operatorname{resp} . \theta_{t}^{A} \tau_{t, k}^{A}+\tau_{t, k}^{B}<P_{t, k}\right)$, then $A$ (resp. $B$ ) will sell back any positive amount of the public good $k$. This implies $Q_{t, k}^{A}=0$ (resp. $\left.Q_{t, k}^{B}=0\right)$ and, thus, $Q_{t, k}^{B}=Q_{t, k}\left(\right.$ resp. $\left.Q_{t, k}^{A}=Q_{t, k}\right)$.

Testing and recovery. As argued in Appendix B, the revealed preference conditions in Proposition 3 can be reformulated in mixed integer programming (MIP) terms. This complements existing MIP characterizations of the cooperative model (in Cherchye, De Rock and Vermeulen (2011)) and the noncooperative model without caring (in Cherchye, Demuynck and De Rock (2011)). The attractive feature of the MIP characterization is that it allows for checking consistency of a given data $S$ with the conditions in Proposition 3. In the spirit of Varian (1982), we refer to this as 'testing' data consistency with the model under study.

More specifically, all constraints of the MIP formulation are linear for fixed $\theta_{t}^{A}$ and $\theta_{t}^{B}$ (see Appendix B). Linearity implies that the above program can be solved by standard MIP methods for a given data set $S$. If we do not know the values of $\theta_{t}^{A}$ and $\theta_{t}^{B}$ (which is usually the case), then we suggest to conduct a grid search that checks the above problem (through MIP methods) for a whole range of possible values for $\theta_{t}^{A}$ and $\theta_{t}^{B}$. For example, in our empirical application we will use an equally sparse grid search with step 0.01.

If observed behavior is consistent with our model with caring (i.e. the set $S$ is rationalizable with caring), then a natural next question pertains to recovering/identifying structural features of the decision model that underlies the (rationalizable) observed consumption behavior. In our application, we will illustrate recovery/identification of values for $\theta$ that are consistent with a rationalization of a given set $S$. Given our discussion in the preceding sections, this value can be interpreted in terms of intra-group cooperation (or caring) that is revealed in the observed consumption behavior. Other recovery questions may pertain to the MWTP values $\tau_{t, k}^{M}\left(q^{M}, Q\right)$ and individual income shares $Y_{t}^{M}$ at equilibrium (in terms of the model with transfers; see Definition 2). Generally, such recovery can start from the MIP methodology presented in this paper. In this respect, we can refer to Cherchye, De Rock and Vermeulen (2011), who consider these questions for the cooperative model; their analysis is directly extended to the noncooperative model with caring discussed here. These authors' basic argument is that revealed preference recovery on the basis of an MIP 
characterization of rational behavior boils down to defining feasible sets characterized by the MIP constraints.

As for recovery of the individual income shares, one important final remark pertains to restrictions S.4 and S.5 in Proposition 3. As we will explain below, these restrictions imply that the shares $Y_{t}^{A}$ and $Y_{t}^{B}$ that underlie observed (rationalizable) behavior are not identifiable in general. This contrasts with the cooperative case in which the withingroup income distribution (in general) can be identified from the observed set $S$. This identifiability result does not generally hold under noncooperative behavior with caring. As a matter of fact, this identifiability problem for our model actually parallels a similar problem for the noncooperative model without caring. ${ }^{16}$

To see the identifiability problem, we first note that the budget constraints in DOP-A and DOP-B imply

$$
\begin{aligned}
& p_{t}^{\prime} q_{t}^{A}+\left(P_{t}-\theta_{t}^{B} \tau_{t}^{B}\right)^{\prime} Q_{t}^{A}+\theta_{t}^{A} \tau_{t}^{A \prime} Q_{t}^{B}=Y_{t}^{A}, \text { and } \\
& p_{t}^{\prime} q_{t}^{B}+\left(P_{t}-\theta_{t}^{A} \tau_{t}^{A}\right)^{\prime} Q_{t}^{B}+\theta_{t}^{B} \tau_{t}^{B \prime} Q_{t}^{A}=Y_{t}^{B} .
\end{aligned}
$$

Thus, because of conditions S.4 and S.5 we obtain that $Y_{t}^{A}$ and $Y_{t}^{B}$ are uniquely identified only if we have, for all $k$ and $t$,

$$
\tau_{t, k}^{A}+\theta_{t}^{B} \tau_{t, k}^{B}<P_{t, k}
$$

so that $Q_{t, k}^{A}=0$ and $Q_{t, k}^{B}=Q_{t, k}$, or

$$
\tau_{t, k}^{B}+\theta_{t}^{A} \tau_{t, k}^{A}<P_{t, k}
$$

so that $Q_{t, k}^{B}=0$ and $Q_{t, k}^{A}=Q_{t, k}$. In terms of the noncooperative model without caring, this last situation would conform to the so-called separate spheres concept. ${ }^{17}$

On the other hand, as soon as there is one public good $k$ to which both individuals contribute for some $t$, i.e.

$$
\tau_{t, k}^{A}+\theta_{t}^{B} \tau_{t, k}^{B}=\tau_{t, k}^{B}+\theta_{t}^{A} \tau_{t, k}^{A}=P_{t, k},
$$

it is impossible to exactly recover the income shares $Y_{t}^{A}$ and $Y_{t}^{B}$ that are consistent with a rationalization of the given data. Specifically, in this case $Q_{t, k}^{A}$ and $Q_{t, k}^{B}$ can take any value (under the sole condition $Q_{t, k}^{A}+Q_{t, k}^{B}=Q_{t, k}$ ) and, thus, the expenditures on good $k$ cannot be assigned to the individual group members. Interestingly, this last result complies with the so-called local income pooling result for the noncooperative model without caring. ${ }^{18}$

\footnotetext{
${ }^{16}$ See Cherchye, Demuynck and De Rock (2011) for more discussion on the identification of individual income shares on the basis of testable revealed preference conditions for the noncooperative model without caring.

${ }^{17}$ See, for example, Lundberg and Pollak (1993) and Browning, Chiappori and Lechene (2010).

${ }^{18}$ See, for example, Kemp (1984), Bergstrom, Blume and Varian (1986) and Browning, Chiappori and Lechene (2010). Importantly, even though we cannot identify $Y_{t}^{A}$ and $Y_{t}^{B}$ under jointly contributed public goods, it is still possible to recover upper and lower bounds on values for $Y_{t}^{A}$ and $Y_{t}^{B}$ that are consistent with a rationalization with caring of the given data set. These bounds then account for the total (nonassignable) expenditures on the jointly contributed public goods.
} 
Model fit. The above conditions for rationalizable behavior imply 'exact' tests: data either satisfy the GARP requirements in Proposition 3 or not. In our empirical application, we will use an index to assess how closely behavior complies with rationalizability. This index will serve as a 'goodness-of-fit' measure that has a specific interpretation as capturing the 'economic significance' of violations of our rationalizability conditions. Specifically, the index calculates the minimal amount by which individual budget constraints must be shifted downward in order to remove all violations of the individual GARP conditions. In this sense, it is similar in spirit to the GARP-based goodness-of-fit measures that have been proposed by Afriat (1973) and Varian (1990; see also our discussion below). ${ }^{19}$ To formally define our index, we use the following extension of Definition 4.

Definition 5 Consider a set $Z=\left\{w_{l}, x_{l}\right\}_{l \in L}$ and a set of non-negative numbers $\left\{c_{l}\right\}_{l \in L}$. For any $l_{1}, l_{2} \in L$, define $x_{l_{1}} \widetilde{R^{D}} x_{l_{2}}$ if

$$
w_{l_{1}}^{\prime} x_{l_{1}}-c_{l_{1}} \geq w_{l_{1}}^{\prime} x_{l_{2}} .
$$

Next, $x_{l_{1}} \widetilde{R} x_{l_{2}}$ if there exists a sequence $r, \ldots, t$ (with $r, \ldots, t \in L$ ) such that

$$
x_{l_{1}} \widetilde{R^{D}} x_{r}, \ldots, x_{t} \widetilde{R^{D}} x_{l_{2}}
$$

Conditional on $\left\{c_{l}\right\}_{l \in L}$, the set $Z$ satisfies $G A R P$ if, for all $l_{1}, l_{2} \in L, x_{l_{1}} \widetilde{R} x_{l_{2}}$ implies

$$
w_{l_{2}}^{\prime} x_{l_{1}} \geq w_{l_{2}}^{\prime} x_{l_{2}}-c_{l_{2}} \text {. }
$$

The adjusted revealed preference relation $\widetilde{R^{D}}$ weakens the revealed preference relation $R^{D}$ (i.e. $w_{l_{1}} x_{l_{1}} \geq w_{l_{1}} x_{l_{2}}$ ) by allowing individuals to waste an amount of $c_{l_{1}}$ of the budget. The usual GARP condition in Definition 4 complies with $c_{l}=0$ for all $l \in L$. Generally, higher values of $c_{l}$ imply weaker rationalizability restrictions.

For a given set $Z$, let

$$
f=\sum_{l} c_{l}
$$

Then, our goodness-of-fit index $f^{*}$ equals the smallest value of $f$ that makes the set $Z$ satisfy the GARP condition in Definition 5. Intuitively, the index provides a measure of how much we have to perturb the budgets to satisfy the revealed preference restrictions.

As a final note, we point out the formal analogy between our goodness-of-fit index and the index proposed by Varian (1990). Instead of using the revealed preference relation $\widetilde{R^{D}}$ in Definition 5, Varian's index is based on an adjusted relation $\overline{R^{D}}$ such that $x_{l_{1}} \overline{R^{D}} x_{l_{2}}$ if

$$
\left(1-e_{l_{1}}\right) w_{l_{1}} x_{l_{1}} \geq w_{l_{1}} x_{l_{2}},
$$

where each factor $e_{l}$ is a number between 0 and 1 . The relation $\widetilde{R^{D}}$ coincides with Varian's relation $\overline{R^{D}}$ if we define

$$
c_{l_{1}}=e_{l_{1}} w_{l_{1}} x_{l_{1}} .
$$

\footnotetext{
${ }^{19}$ For more details (including applications) of the indices suggested by Afriat (1973) and Varian (1990), we refer to the recent discussions of Choi, Kariv, Muller and Silverman (2014) and Halevy, Persitz and Zrill (2016).
} 
Essentially, our index evaluates goodness-of-fit in terms of absolute budget shifts, while Varian's index uses relative shifts. Our main motivation to focus on absolute (and not relative) shifts is of a computational nature. For the rationalizability conditions in Proposition 3 , using our (absolute) index $f^{*}$ obtains a mix integer programming (MIP) problem that is linear in unknowns (see Appendix C). By contrast, using a Varian-type (relative) index implies constraints that are quadractic in unknowns, which are substantially more complex to handle. ${ }^{20}$ In this respect, it is worth remarking that in our following application the budget levels are held constant over all decision situations. In this specific setting, the use of absolute versus relative budget shifts is obviously irrelevant for comparing these shifts over decision situations.

\section{Application: joint decisions of children}

To show the practical usefulness of our model, we apply it to analyze consumption decisions made by dyads of children in an experimental setting. This empirical application fits within the growing interest in understanding children's economic rationality (see, for example, Harbaugh, Krause and Berry, 2002, and Seguin, Arseneault and Tremblay, 2007) and (group) decision-making (recent examples include Fehr, Glätzle-Rützler and Sutter, 2013, Angerer, Glätzle-Rützler, Lergetporer and Sutter, 2016, and Czermak, Feri, Glätzle-Rützler and Sutter, 2016). This gained interest is motivated by children's increasing economic importance, in terms of their impact on joint decisions with parents (see, for example, Calvert, 2008) and peers (see, for example, Wouters, Larsen, Kremers, Dagnelie and Geenen, 2010). Because observational data on joint decisions made by children are typically not available, we make use of a laboratory experiment that is specially tailored to obtain the data needed for our revealed preference methodology.

As we explain in more detail below, our following empirical exercise uses an experimental design that is closely similar to the one of Bruyneel, Cherchye, Cosaert, De Rock and Dewitte (2017). A main difference with this earlier study is that our current design uses consumption bundles that contain a public good. This makes it possible to empirically distinguish between models with different degrees of caring on the basis of our characterization in Proposition 3. In addition, an advantage of our experimental design is that we not only observe joint choices of the children dyads but also the children's individual choices. This gives us additional identifying information on the individual preferences, which benefits our testing analysis as well as our recovery results on the degree of within-dyad cooperation.

After presenting our sample and the specificities of our experimental design, we will first demonstrate that the noncooperative model with caring effectively does provide an empirically relevant extension of the fully cooperative and noncooperative (without caring) models. In particular, our caring model will achieve a better 'goodness-of-fit' than these other models. Second, we will show that we can informatively (set) identify the degree of caring for individual dyads by using our revealed preference methodology. We obtain upper and lower bounds on the caring parameter that are very tight (close to point identification)

\footnotetext{
${ }^{20}$ Technically, when starting from the rationalizability conditions in Proposition 3, the rationalizability constraints will become quadratic because the factors $e_{l}$ (defined above) have to be multiplied with unknowns representing the individuals' willingness to pay for the observed consumption quantities.
} 


\begin{tabular}{llll}
\hline & obs & mean & std dev \\
\hline third grade & 61 & 0.295 & 0.460 \\
sixth grade & 61 & 0.295 & 0.460 \\
social (dyad mean) & 58 & 6.816 & 1.084 \\
assertive (dyad mean) & 58 & 6.578 & 1.225 \\
interaction & 61 & 0.295 & 0.460 \\
mixed & 61 & 0.475 & 0.504 \\
both female & 61 & 0.262 & 0.444 \\
\hline
\end{tabular}

Table 1: Summary statistics of observed dyad characteristics

for a significant number of dyads. This demonstrates that the caring model can have substantial empirical bite, even when only exploiting its nonparametric testable implications. Finally, we will explore to what extent the observed variation in caring across dyads can be related to observed dyad characteristics.

To facilitate the following discussion, we will focus on the caring model with the degree of intra-dyad cooperation held constant over all observed decision situations, i.e. we consider $\theta_{t}^{J}=\theta^{J}$ for all $t$ and $J \in\{A, B\}$. The underlying assumption is that the degree of intra-dyad cooperation does not change over the observations. In this respect, we recall that the fully cooperative model and the noncooperative model without caring correspond to $\theta^{A}=\theta^{B}=1$ and $\theta^{A}=\theta^{B}=0$, respectively. Furthermore, because we found that allowing for variation in caring across dyad members does not increase the fit of the caring model (results available upon request), we will abstract from intra-dyad variation in caring, i.e. we use $\theta^{A}=\theta^{B}=\theta$. In other words, we will specifically focus on dyad-level degrees of cooperation; we will then also relate these dyad-level estimates of $\theta$ to observed dyad characteristics.

Sample and experimental design. We collected our data on children's consumption choices in two different schools in Belgium among three age categories: kindergarten level, third grade and sixth grade. Dropping children with misreported values (18 in total) leads to a sample of 122 participants. The final sample consists of 50 kindergarten children (5-6 years old), 36 third graders (8-9 years old) and 36 sixth graders (11-12 years old). The children were randomly teamed up in pairs conditional on these age brackets. This guarantees sufficient variation in the level of friendship between group members. We also collected information on children's social skills and assertiveness (scale from 1-10) and whether the children in the dyad interacted a lot in the school environment (dummy). This information was obtained from teacher-based assessments. We show summary statistics of these covariates in Table 1.

As indicated above, our experimental design mainly follows the one of Bruyneel, Cherchye, Cosaert, De Rock and Dewitte (2017). Therefore we only briefly summarize our set-up below, and we refer to these authors for more details on the choice sets, experimental instructions and survey questions. We invited the dyads to jointly choose a se- 


\begin{tabular}{rrr}
\hline movie & mandarins & biscuits \\
\hline 16 & 4 & 1 \\
16 & 3 & 2 \\
18 & 3 & 1 \\
2 & 8 & 4 \\
4 & 8 & 3 \\
2 & 9 & 3 \\
8 & 1 & 8 \\
6 & 2 & 8 \\
6 & 1 & 9 \\
\hline
\end{tabular}

Table 2: Implicit prices for the various choice problems.

ries of consumption bundles composed of three non-durable commodities: time to watch movies, mandarins and letter biscuits. The children are faced with nine different choice sets containing seven alternatives. Each choice set corresponds to a unique combination of (implicit) prices (reported in Table 2) and a fixed budget of 24. Following the argument of Harbaugh, Krause and Berry (2001), the discrete nature of the choice sets considerably simplifies the decision problems faced by the children. Each choice set contains three bundles in which the total (implicit) budget is allocated to a single commodity, three bundles in which the budget is spent on two of the three commodities, and one bundle with strictly positive amounts of all three commodities. The children within each dyad are also asked to distribute the mandarins and letter biscuits from the jointly selected bundle among each other, which allows us to perfectly observe the allocation of these goods to each individual member. Strictly speaking, this extra information is not needed to implement our revealed preference conditions. However, it does add considerable empirical bite to our analysis. Finally, the children are clearly informed that they will receive one of their choices at the end of the experiment, which is meant to incentivize them to truly reveal their preferences.

Next, we want to emphasize two specific features of our design, which directly relate to the research question that we study here. A first specificity is that we let children choose between units of movie watching ( 5 minutes per unit), units of mandarins (12.5 gram per unit) and letter biscuits ( 5 gram per unit). The first good gives the children the possibility to jointly watch part of a popular Belgian TV series. ${ }^{21}$ Clearly, watching movies is non-rivalrous and non-exclusive within a given dyad, which makes this a public good. This contrasts with Bruyneel, Cherchye, Cosaert, De Rock and Dewitte (2017), who used grapes instead of movies for the first good, so that all goods were private by construction. Table 2 presents the implicit prices of the goods that we used in each of our nine choice regimes.

The second specific feature of our design is that we not only let the children choose jointly but also individually. More specifically, prior to making the joint decision, the children were invited to make nine individual choices. These individual choices had the same

\footnotetext{
${ }^{21}$ The particular TV series was differentiated in order to appeal to children in the various age brackets.
} 


\begin{tabular}{lcccccc}
\hline & obs & mean & std dev & median & $\min$ & $\max$ \\
\hline movie & 61 & 0.443 & 0.223 & 0.444 & 0 & 1.000 \\
mandarins & 61 & 0.231 & 0.169 & 0.203 & 0 & 0.703 \\
biscuits & 61 & 0.326 & 0.160 & 0.315 & 0 & 0.703 \\
& \multicolumn{7}{c}{ individual experiment } \\
movie & 122 & 0.447 & 0.220 & 0.463 & 0 & 0.917 \\
mandarins & 122 & 0.224 & 0.163 & 0.213 & 0 & 0.694 \\
biscuits & 122 & 0.329 & 0.132 & 0.333 & 0.028 & 0.559 \\
& 61 & -0.004 & 0.136 & -0.019 & -0.352 & 0.361 \\
\hline
\end{tabular}

Table 3: Budget shares chosen in the joint and invidual experiments, and the associated difference in budget share spent on movies

choice sets as in joint decision problems (explained above) except that the (implicit) price of the public good and the individual's (implicit) budgets were halved. These modifications guarantee that, in principle, each child could select exactly the same bundles as in the joint choice problems.

An important advantage of combining choices of the same child from the individual and joint choice settings is that it substantially improves the empirical bite of the revealed preference methods. In the individual choice setting, watching film is a private good. Therefore, in this case, the marginal willingness to pay for the film equals the known (implicit) price. For the joint choice problems, individual marginal willingness to pay for the public good, i.e. $\tau_{t}^{A}$ and $\tau_{t}^{B}$, are unknown but need to satisfy the condition specified in Proposition 3. Under the assumption that each child's preferences remain the same in the individual and joint choice settings, we can use the observed individual choices to improve the bounds on $\tau_{t}^{A}$ and $\tau_{t}^{B}$. This in turn enhances the discriminatory power of our revealed preference tests and the identification of the caring parameter $\theta$.

Table 3 presents summary statistics of the observed budget shares. Average budget shares in the joint choice experiment vary between 0.231 and 0.443 . This indicates that all goods were sufficiently attractive. The mean budget shares for the individual choices are very similar. The bottom line of Table 3 gives summary statistics for the difference between the budget share allocated to film for the individual and joint choice settings. While this difference is fairly small on average, it goes up to no less than 36 percentage points for at least one dyad.

Model fit. We start by testing the revealed preference restrictions of the caring model. For alternative specifications of the caring parameter $\theta$, we evaluate the fit of the associated joint consumption model by computing our goodness-of-fit index $f^{*}$ for every distinct dyad. 
To recall, a lower value for $f^{*}$ indicates a better fit of the observed behavior with the model's assumptions. In Appendix C, we explain our method to compute our index based on the rationalizability conditions in Proposition 3, while accounting for the particularities of our empirical application.

As explained above, the noncooperative model with caring nests the fully cooperative and fully noncooperative (without caring) models. The relevance of the former model thus depends on the extent to which it allows us to better explain the observed choice behavior. Let $f_{\text {caring }}^{*}, f_{\text {coop }}^{*}$ and $f_{\text {noncoop }}^{*}$ represent the value of the indices conditional on whether $\theta \in[0,1]$ (i.e. caring model), $\theta=1$ (i.e. cooperative model) or $\theta=0$ (i.e. noncooperative model), respectively. Table 4 presents summary statistics on the distribution of these three indices. For the full sample of 61 dyads, the mean index values are 5.50, 5.88 and 6.04 respectively. Given the construction of our goodness-of-fit measure (explained in Appendix C), these values can be interpreted as the dyads' total budget waste summed over their different choice experiments. The total available budget over all these choice problems equals 432 (i.e. $2 \cdot 9 \cdot 24$ ). As our mean values for $f_{\text {caring }}^{*}, f_{\text {coop }}^{*}$ and $f_{\text {noncoop }}^{*}$ are small relative to this total budget, we may conclude that all three models fit the data fairly well at the overall sample level.

To further motivate this conclusion, we simulate random data sets by uniformly drawing 1000 bundles from the respective choice sets. For this simulated random choice behavior, we obtain average values for $f_{\text {caring }}^{*}=25.55, f_{\text {coop }}^{*}=26.35$ and $f_{\text {noncoop }}^{*}=26.23$, which are substantially above the averages that are reported in Table 4. Perhaps even more striking, the 10-th percentiles of $f_{\text {caring }}^{*}, f_{\text {coop }}^{*}$ and $f_{\text {noncoop }}^{*}$ for random data sets $(8.79,9.29$ and 9.62 respectively) are still above the mean (and median) values reported in Table 4.

Importantly, Table 4 reveals that the caring model generally provides a better empirical fit of the observed dyads' choice behavior than the fully cooperative and noncooperative models. The question remains whether these differences are statistically significant. To investigate this, we conduct the Wilcoxon matched-pairs signed-ranks test and the Sign test of matched pairs. The first test checks the hypothesis that the distributions of $f_{\text {caring }}^{*}, f_{\text {coop }}^{*}$ and $f_{\text {noncoop }}^{*}$ are the same, while the second test verifies the hypothesis of equal median values. Interestingly, both tests support the same conclusions. First, we can reject the hypotheses that distributions and medians are the same for $f_{\text {caring }}^{*}$ and $f_{\text {coop }}^{*}$ as well for $f_{\text {caring }}^{*}$ and $f_{\text {noncoop }}^{*}$ (both p-values are below 0.0001). By contrast, we are unable to reject the hypotheses of equal distributions and medians for $f_{\text {coop }}^{*}$ and $f_{\text {noncoop }}^{*}$ (the p-value for Wilcoxon test is 0.890 and the p-value for the Sign test is 0.766), which signals that the noncooperative model does not significantly outperform the cooperative model or vice versa. In our opinion, these results clearly motivate the empirical relevance of our newly proposed caring model in terms of explaining the observed group consumption behavior.

Identifying the degree of caring. By using the revealed preference restrictions outlined in Section 4, we can identify upper and lower bounds on the caring parameter $\theta$ that are consistent with rationalizing the observed choice behavior. This effectively obtains 'set' identification (to be contrasted with more standard 'point' identification) of this parameter. 


\begin{tabular}{lllllll}
\hline & obs & mean & std dev & median & $\min$ & $\max$ \\
\hline$f_{\text {caring }}^{*}$ & 61 & 5.503 & 8.605 & 0.600 & 0 & 38.711 \\
$f_{\text {coop }}^{*}$ & 61 & 5.879 & 8.785 & 1.313 & 0 & 39.031 \\
$f_{\text {noncoop }}^{*}$ & 61 & 6.043 & 9.018 & 1.200 & 0 & 38.711 \\
\hline
\end{tabular}

Table 4: Summary statistics of the goodness-of-fit index.

\begin{tabular}{lcccccc}
\hline & obs & mean & std dev & median & $\min$ & $\max$ \\
\hline$\theta^{l b}$ & 61 & 0.211 & 0.372 & 0 & 0 & 1 \\
$\theta^{u b}$ & 61 & 0.820 & 0.344 & 1 & 0 & 1 \\
$\bar{\theta}$ & 61 & 0.515 & 0.284 & 0.5 & 0 & 1 \\
\hline
\end{tabular}

Table 5: Summary statistics of $\theta^{l b}, \theta^{u b}$ and $\bar{\theta}$

Such set identification is standard in revealed preference analysis. ${ }^{22}$ More specifically, for each dyad we obtain the lower bound $\theta^{l b}$ on the caring parameter by minimizing $\theta$ subject to the rationalizability restrictions in Proposition 3 , while fixing our index $f_{\text {caring }}^{*}$ at its value computed above. Analogously, we compute the upper bound $\theta^{u b}$ by maximizing $\theta$ subject to the same restrictions.

Table 5 presents summary statistics for $\theta^{l b}, \theta^{u b}$ and the midpoint of the interval $\left[\theta^{l b}, \theta^{u b}\right]$, denoted by $\bar{\theta}$. We find that the average lower bound is 0.211 , which is substantially above the zero value that applies to the noncooperative model without caring. Similarly, the average upper bound equals 0.820 , which is significantly below the value of unity that holds for the fully cooperative model. Further, we observe that the average value of $\bar{\theta}$ amounts to 0.515 . These results again show the value added of our caring model relative to the cooperative and noncooperative models in terms of explaining the observed dyad behavior. In addition, the standard deviations and the minimum and maximum values that are reported in Table 5 suggest quite some variation in the degree of caring across dyads. In this respect we also note that the minimal upper bound equals zero and the maximal lower bound equals one.

Next, Table 6 provides a summary of the distribution of $\Delta=\theta^{u b}-\theta^{l b}$ over the 61 dyads. We obtain $\theta^{u b} \approx \theta^{l b}$ for 14 dyads, which shows that the identification can be very precise (i.e. close to point identification). On the other hand, for 26 of the 61 dyads, we get $\Delta=1$, which corresponds to $\theta^{l b}=0$ and $\theta^{u b}=1$. For these dyads, our rationalizability restrictions do not reveal specific information on the value of $\theta$.

As a final exercise, we link the identified variation in the caring parameter to observed dyad characteristics. In our regressions, we use the maximum degree of caring $\left(\theta^{u b}\right)$ as

\footnotetext{
${ }^{22}$ See, for example, Adams (2015) and Cherchye, Demuynck, De Rock and Vermeulen (2017) for more discussion and recent examples.
} 


\begin{tabular}{ll}
$\Delta=\theta^{u b}-\theta^{l b}$ & obs \\
\hline$\approx 0$ & 14 \\
$\leq 0.25$ & 19 \\
$\leq 0.5$ & 25 \\
$\leq 0.75$ & 29 \\
$<1$ & 35 \\
$=1$ & 26 \\
\hline
\end{tabular}

Table 6: Distribution of $\Delta=\theta^{u b}-\theta^{l b}$

dependent variable. Our motivation is two-fold. First, the value of $\theta^{u b}$ varies substantially over our sample of dyads, which obviously is useful for an informative explanatory analysis. Second, and more importantly, the upper bound $\theta^{u b}$ has an intuitive interpretation as quantifying the minimum deviation from the fully cooperative model that is required to rationalize the observed dyad behavior. Given its popularity in the applied literature on group/household consumption, this cooperative model constitutes a natural benchmark model of group behavior. This makes it particularly interesting to empirically investigate which dyad characteristics correspond to more or less behavioral consistency with the model (captured by $\theta^{u b}$ ). We include seven dyad characteristics as covariates in our regressions: a dummy for third grade, a dummy for sixth grade, a dummy indicating whether the dyad members interact strongly in the daily school environment, two teacher-based measures of social skills and assertiveness, and two gender covariates showing whether the dyad is mixed or consists of two females. Due to missing observations on some of these covariates, we had to remove 3 dyads in our regressions, yielding a sample with 58 dyads.

Table 7 presents our results. In the first regression specification, we include all dyads of our sample, thus also the ones with high levels of $f_{\text {caring }}^{*}$ (i.e. low goodness-of-fit). The first two columns of Table 7 report our findings from a simple OLS regression and, respectively, Papke and Wooldridge (1996)'s quasi-maximum likelihood estimation procedure for fractional response data (which accounts for $\theta^{u b}$ situated between 0 and 1 with many observations equal to 0 or 1 ). Even though our sample size is small, we do observe that the dummy for interaction is strongly significant (at the $5 \%$ level), while our measure of assertiveness is borderline significant in the OLS regression. The regressions do not show an (additional) significant effect of our social skills measure.

As a robustness check, we also consider a second specification, for which we exclude the 6 dyads of which the value for $f_{\text {caring }}^{*}$ is situated above the 90 -th percentile for our sample. The value of this 90 -th percentile equals 18 , which is about 4.2 percent of a dyad's total available budget ( $=432$, as explained above). Thus, in terms of our goodness-of-fit measure, the behavior of the selected 52 dyads is reasonably close to that predicted by the caring model, as low levels of $f^{*}$ signal a high degree of consistency with the caring model. The results of these additional regressions are given in the third and fourth columns of Table 7. Interestingly, our qualitative conclusions are the same as before. Again, dyads who 


\begin{tabular}{lcccc}
\hline$\theta^{u b}$ & \multicolumn{2}{c}{ all } & \multicolumn{2}{c}{$90 \%$-lowest } \\
& OLS & $Q M L$ & $O L S$ & $Q M L$ \\
\hline third grade & 0.035 & 0.257 & -0.031 & -0.333 \\
& $(0.105)$ & $(0.805)$ & $(0.102)$ & $(0.937)$ \\
sixth grade & -0.080 & -0.487 & -0.184 & -1.424 \\
& $(0.109)$ & $(0.825)$ & $(0.110)$ & $(0.900)$ \\
social & -0.061 & -0.393 & -0.076 & -0.463 \\
& $(0.051)$ & $(0.353)$ & $(0.049)$ & $(0.346)$ \\
assertive & $0.078^{*}$ & 0.509 & $0.087^{* *}$ & 0.584 \\
& $(0.046)$ & $(0.407)$ & $(0.043)$ & $(0.360)$ \\
interaction & $0.208^{*}$ & $2.055^{* *}$ & $0.190^{*}$ & $1.970^{* *}$ \\
& $(0.105)$ & $(0.844)$ & $(0.102)$ & $(0.955)$ \\
mixed & 0.085 & 0.585 & 0.054 & 0.261 \\
& $(0.111)$ & $(0.878)$ & $(0.113)$ & $(1.146)$ \\
female & -0.053 & -0.368 & -0.092 & -0.873 \\
& $(0.123)$ & $(0.963)$ & $(0.123)$ & $(1.187)$ \\
intercept & $0.658^{* *}$ & 0.533 & $0.794^{* *}$ & 1.476 \\
& $(0.316)$ & $(2.838)$ & $(0.311)$ & $(2.765)$ \\
\hline obs & 58 & 58 & 52 & 52 \\
$R^{2}$ & 0.148 & & 0.204 & \\
\hline
\end{tabular}

Table 7: Regression results (* and ${ }^{* *}$ indicating statistical significance at 10 percent and 5 percent, respectively)

strongly interact have a larger value of the caring parameter $\theta^{u b}$. Also, the OLS regression indicates that the coefficient for assertiveness is positively related to the estimate of $\theta^{u b}$. Dyads with more assertive members may be more inclined to share information on their preferences and may also be more eager to interact in order to reach a more cooperative solution. The finding that more assertive and interacting dyads behave more cooperatively confirms our prior intuition. In our opinion, these findings provide additional validation to the empirical usefulness of our caring model and our proposed measure for the degree of cooperation.

\section{Conclusion}

We have presented a model for analyzing group consumption behavior that simultaneously accounts for caring preferences and noncooperative behavior. Interestingly, by varying the degree of intra-group caring, the model encompasses a whole continuum of consumption models situated between the fully cooperative model (assuming a Pareto efficient outcome) and the noncooperative model without caring (assuming a public good game with voluntary 
contributions). Attractively, our newly proposed model also allowed us to define a measure for the degree of intra-group cooperation, which quantifies how close the observed group behavior is to the fully cooperative benchmark. Following a revealed preference approach, we derived the testable implications of the model for empirical data.

We demonstrated the empirical relevance of our theoretical model through an empirical application to consumption choices made by dyads of children. We found that our new caring model provided a better fit of the data compared to the fully cooperative and noncooperative models. Next, we showed that we can informatively identify the degree of intra-group caring by using our nonparametric revealed preference methodology. Our results suggest that cooperation varies considerably across dyads, and that the degree of cooperation is often situated strictly between the extreme cases of full cooperation and noncooperation without any caring. We also found that our measure of cooperation is positively correlated with assertiveness and the degree of interaction within dyads.

We believe that these empirical results validate the practical usefulness of our consumption model to investigate cooperation in group consumption, as well as its defining characteristics. While we used experimental data in the current study, our revealed preference methodology can also be used in combination with observational data. For example, an interesting application may identify the degree of cooperation in household consumption, and relate inter-household heterogeneity to specific household (member) characteristics. In this respect, we can also refer to Cherchye, De Rock and Vermeulen $(2009,2011)$ and Cherchye, Demuynck and De Rock (2011) for empirical studies of household consumption behavior that make use of revealed preference methods similar to ours.

\section{A Proofs}

\section{A.1 Proof of Proposition 1}

The first order conditions for OP-A and OP-B with respect to the numeraire (i.e. the first private good) and public goods $k$ are

$$
\begin{aligned}
& \frac{\partial W_{t}^{A}}{\partial U^{A}} \frac{\partial U^{A}}{\partial q_{1}^{A}} \leq \lambda_{t}^{A}, \\
& \frac{\partial W_{t}^{B}}{\partial U^{A}} \frac{\partial U^{A}}{\partial q_{1}^{A}} \leq \lambda_{t}^{B}, \\
& \frac{\partial W_{t}^{A}}{\partial U^{B}} \frac{\partial U^{B}}{\partial q_{1}^{B}} \leq \lambda_{t}^{A}, \\
& \frac{\partial W_{t}^{B}}{\partial U^{B}} \frac{\partial U^{B}}{\partial q_{1}^{B}} \leq \lambda_{t}^{B}, \\
& \frac{\partial W_{t}^{A}}{\partial U^{A}} \frac{\partial U^{A}}{\partial Q_{k}}+\frac{\partial W_{t}^{A}}{\partial U^{B}} \frac{\partial U^{B}}{\partial Q_{k}} \leq \lambda_{t}^{A} P_{t, k}, \\
& \frac{\partial W_{t}^{B}}{\partial U^{A}} \frac{\partial U^{A}}{\partial Q_{k}}+\frac{\partial W_{t}^{B}}{\partial U^{B}} \frac{\partial U^{B}}{\partial Q_{k}} \leq \lambda_{t}^{B} P_{t, k},
\end{aligned}
$$


with $\lambda_{t}^{A}$ and $\lambda_{t}^{B}$ the Lagrange multipliers of the respective budget constraints. We start from the following observations:

- Either (1) or (2) must hold with equality. This follows from the fact that $q_{t, 1}^{A}$ is strictly positive.

- Either (3) or (4) must hold with equality. This follows from the fact that $q_{t, 1}^{B}$ is strictly positive.

- Either (5) or (6) must hold with equality. This follows from the fact that $Q_{t, k}$ is strictly positive.

- Not both (1) and (4) have strict inequality.

Proof. We prove ad absurdum. Suppose both (1) and (4) hold with strict inequality, then by the first two observations above, it must be that (2) and (3) hold with equality. Then, dividing condition (1) by (2) gives:

$$
\frac{\frac{\partial W_{t}^{A}}{\partial U^{A}}}{\frac{\partial W_{t}^{B}}{\partial U^{A}}}<\frac{\lambda_{t}^{A}}{\lambda_{t}^{B}},
$$

while dividing (3) by (4) gives:

$$
\frac{\lambda_{t}^{A}}{\lambda_{t}^{B}}<\frac{\frac{\partial W_{t}^{A}}{\partial U^{B}}}{\frac{\partial W_{t}^{B}}{\partial U^{B}}} .
$$

These two inequalities impose that:

$$
-\frac{\frac{\partial W_{t}^{A}}{\partial U^{A}}}{\frac{\partial W_{t}^{A}}{\partial U^{B}}}>-\frac{\frac{\partial W_{t}^{B}}{\partial U^{A}}}{\frac{\partial W_{t}^{B}}{\partial U^{B}}} .
$$

This contradicts Assumption SC.

The above reasoning gives us three possible cases: (i) both (1) and (3) hold with equality, (ii) both (1) and (4) hold with equality, (iii) both (2) and (4) hold with equality.

Case (i) In this case, equation (5) can be rewritten as

$$
\left(\tau_{k}^{A}\left(q_{t}^{A}, Q_{t}\right)+\tau_{k}^{B}\left(q_{t}^{B}, Q_{t}\right)\right) \leq P_{t, k}
$$

Further, we have that,

$$
\begin{aligned}
\frac{\partial W_{t}^{B}}{\partial U^{A}} \frac{\partial U^{A}}{\partial Q_{k}}+\frac{\partial W_{t}^{B}}{\partial U^{B}} \frac{\partial U^{B}}{\partial Q_{k}} & \leq \lambda_{t}^{B}\left(\tau_{k}^{A}\left(q_{t}^{A}, Q_{t}\right)+\tau_{k}^{B}\left(q_{t}^{B}, Q_{t}\right)\right) \\
& \leq \lambda_{t}^{B} P_{t, k}
\end{aligned}
$$


The inequality in (8) follows from using conditions (2) and (4). The inequality in (9) follows from (7).

As one of the two conditions (5) or (6) must hold with equality, we have that that $\tau_{k}^{A}\left(q_{t}^{A}, Q_{t}\right)+\tau_{k}^{B}\left(q_{t}^{B}, Q_{t}\right)=P_{t, k}$. As $k$ was arbitrary, this holds for every public good. Setting $\theta_{t}^{A}=\theta_{t}^{B}=1$ demonstrates the proof.

Case (ii) For this case, we can rewrite conditions (5) and (6) as:

$$
\begin{aligned}
\frac{\partial W_{t}^{A}}{\partial U^{A}} \frac{\partial U^{A}}{\partial Q_{k}}+\frac{\partial W_{t}^{A}}{\partial U^{B}} \frac{\partial U^{B}}{\partial Q_{k}} & =\lambda_{t}^{A} \tau_{k}^{A}\left(q_{t}^{A}, Q_{t}\right)+\frac{\frac{\partial W_{t}^{A}}{\partial U^{B}}}{\frac{\partial W_{t}^{B}}{\partial U^{B}}} \lambda_{t}^{B} \tau_{k}^{B}\left(q_{t}^{B}, Q_{t}\right) \\
& \leq \lambda_{t}^{A} P_{t, k}
\end{aligned}
$$

and,

$$
\begin{aligned}
\frac{\partial W_{t}^{B}}{\partial U^{A}} \frac{\partial U^{A}}{\partial Q_{k}}+\frac{\partial W_{t}^{B}}{\partial U^{B}} \frac{\partial U^{B}}{\partial Q_{k}} & =\frac{\frac{\partial W_{t}^{B}}{\partial U^{A}}}{\frac{\partial W_{t}^{A}}{\partial U^{A}}} \lambda_{t}^{A} \tau_{k}^{A}\left(q_{t}^{A}, Q_{t}\right)+\lambda_{t}^{B} \tau_{k}^{B}\left(q_{t}^{B}, Q_{t}\right) \\
& \leq \lambda_{t}^{B} P_{t, k}
\end{aligned}
$$

As one of these two conditions must hold with equality, we have that:

$$
\max \left\{\begin{array}{l}
\tau_{k}^{A}\left(q_{t}^{A}, Q_{t}\right)+\theta_{t}^{B} \tau_{k}^{B}\left(q_{t}^{B}, Q_{t}\right) ; \\
\tau_{k}^{B}\left(q_{t}^{B}, Q_{t}\right)+\theta_{t}^{A} \tau_{k}^{A}\left(q_{t}^{A}, Q_{t}\right)
\end{array}\right\}=P_{t, k}
$$

where

$$
\theta_{t}^{A}=\frac{\frac{\partial W_{t}^{B}}{\partial U^{A}}}{\frac{\partial W_{t}^{A}}{\partial U^{A}}} \frac{\lambda_{t}^{A}}{\lambda_{t}^{B}} \leq \frac{\lambda_{t}^{B}}{\lambda_{t}^{A}} \frac{\lambda_{t}^{A}}{\lambda_{t}^{B}}=1
$$

and,

$$
\theta_{t}^{B}=\frac{\frac{\partial W_{t}^{A}}{\partial U^{B}}}{\frac{\partial W_{t}^{B}}{\partial U^{B}}} \frac{\lambda_{t}^{B}}{\lambda_{t}^{A}} \leq \frac{\lambda_{t}^{A}}{\lambda_{t}^{B}} \frac{\lambda_{t}^{B}}{\lambda_{t}^{A}}=1
$$

The inequality in (11) follows from dividing condition (2) by (1) while the inequality in (12) follows from dividing condition (3) by (4).

Case(iii) This case is analogous to case (i). 


\section{A.2 Proof of Proposition 2}

\section{A.2.1 Proof of statement 1}

Assume that for each decision situation $t$ we have that $\left\{q_{t}^{A, A}, q_{t}^{A, B}, q_{t}^{B, B}, q_{t}^{B, A}, Q_{t}^{A}, Q_{t}^{B}\right\}$ satisfies the definition of a household equilibrium with caring for the utility functions $U^{A}$, $U^{B}$, caring functions $W_{t}^{A}, W_{t}^{B}$, prices $p_{t}, P_{t}$ and household income $Y_{t}$.

We need to show that there exist numbers $\theta_{t}^{A}, \theta_{t}^{B} \in[0,1]$ and incomes $Y_{t}^{A}, Y_{t}^{B}$ (with $\left.Y_{t}^{A}+Y_{t}^{B}=Y_{t}\right)$ such that $\left\{q_{t}^{A}, q_{t}^{B}, Q_{t}^{A}, Q_{t}^{B}\right\}$ is a household equilibrium with transfers. Let us first focus on individual $A$. For the proof, we will again distinguish three cases, identical to the cases used in the proof of Proposition 1.

Before we begin, consider the first order condition for $A$ and $B$ with respect to the $n$th private good for $A$ (i.e. the quantities $q_{t, n}^{A, A}$ and $q_{t, n}^{B, A}$ ):

$$
\begin{aligned}
& \frac{\partial W_{t}^{A}}{\partial U^{A}} \frac{\partial U^{A}}{\partial q_{n}^{A}} \leq \lambda_{t}^{A} p_{t, n} \\
& \frac{\partial W_{t}^{B}}{\partial U^{A}} \frac{\partial U^{A}}{\partial q_{n}^{A}} \leq \lambda_{t}^{B} p_{t, n}
\end{aligned}
$$

Lemma 1 If case (i) or (ii) holds and $q_{t, n}^{A}>0$, then (13) holds with equality for all private goods $s$ at equilibrium. On the other hand if case (iii) holds and $q_{t, n}^{A}>0$, then (14) holds with equality for all private goods $n$ at equilibrium.

Proof. Assume that either case (i) or (ii) holds and that $\frac{\partial W_{t}^{A}}{\partial U^{A}} \frac{\partial U^{A}}{\partial q_{n}^{A}}<\lambda_{t}^{A} p_{t, n}$. Then, since $q_{t, n}^{A}>0$ it must be that $\frac{\partial W^{B}}{\partial U^{A}} \frac{\partial U^{A}}{\partial q_{n}^{A}}=\lambda_{t}^{B} p_{t, n}$. Dividing these two conditions gives:

$$
\begin{aligned}
& \frac{\frac{\partial W_{t}^{A}}{\partial U^{A}}}{\frac{\partial W_{t}^{B}}{\partial U^{A}}}<\frac{\lambda_{t}^{A}}{\lambda_{t}^{B}} \\
& \frac{\frac{\partial W_{t}^{A}}{\partial U^{A}}}{\frac{\partial W_{t}^{B}}{\partial U^{A}}} \geq \frac{\lambda_{t}^{A}}{\lambda_{t}^{B}},
\end{aligned}
$$

a contradiction. A similar reasoning holds for the second part of the Lemma.

Let us now consider the three relevant cases that were also considered in the proof of Proposition 1:

Case (i) In this case, we set $\theta_{t}^{A}=\theta_{t}^{B}=1$ and we define:

$$
Y_{t}^{A}=p_{t}^{\prime} q_{t}^{A}+\left(P_{t}-\tau_{t}^{B}\right)^{\prime} Q_{t}^{A}+\tau_{t}^{A \prime} Q_{t}^{B}
$$

Towards a contradiction, let us consider an allocation $\left(q^{A}, Q^{A}\right)$ such that

$$
p_{t}^{\prime} q^{A}+\left(P_{t}-\tau_{t}^{B}\right)^{\prime} Q^{A}+\tau_{t}^{A \prime} Q_{t}^{B} \leq Y_{t}^{A}
$$


and,

$$
U^{A}\left(q^{A}, Q^{A}+Q_{t}^{B}\right)>U^{A}\left(q_{t}^{A}, Q_{t}^{A}+Q_{t}^{B}\right) .
$$

Denote by $U_{q_{t}^{A}}^{A}$ and $U_{Q_{t}}^{A}$ the subgradient vectors for $U^{A}$ with respect to $q^{A}$ and $Q$ at the bundles $\left(q_{t}^{A}, Q_{t}\right)$. Then, by concavity of $U^{A}$, we have that:

$$
\begin{aligned}
U^{A}\left(q^{A}, Q^{A}+Q_{t}^{B}\right)-U^{A}\left(q_{t}^{A}, Q_{t}^{A}+Q_{t}^{B}\right) & \leq U_{q_{t}^{A}}^{A}\left(q^{A}-q_{t}^{A}\right)+U_{Q_{t}}^{A}\left(Q^{A}-Q_{t}^{A}\right) \\
& =\frac{\lambda_{t}^{A}}{\frac{\partial W_{t}^{A}}{\partial U_{t}^{A}}}\left[p_{t}^{\prime}\left(q^{A}-q_{t}^{A}\right)+\left(P_{t}-\tau_{t}^{B}\right)^{\prime}\left(Q^{A}-Q_{t}^{A}\right)\right] \\
& \leq 0 .
\end{aligned}
$$

The first inequality follows from Lemma 1 and the fact that condition (5) must hold with equality for case (i). The second inequality follows from the budget constraint and gives us the desired contradiction.

Case (ii) In this case, we define $\theta_{t}^{B}$ and $\theta_{t}^{A}$ as in conditions (11) and (12) and we define $Y_{t}^{A}$ by

$$
Y_{t}^{A}=p_{t}^{\prime} q_{t}^{A}+\left(P_{t}-\theta_{t}^{B} \tau_{t}^{B}\right)^{\prime} Q_{t}^{A}+\theta_{t}^{A} \tau_{t}^{A \prime} Q_{t}^{B}
$$

One can easily see that for case (ii), $Q_{t, k}^{A}>0$ implies that $\tau_{k}^{A}\left(q_{t}^{A}, Q_{t}\right)+\theta_{t}^{B} \tau_{k}^{B}\left(q_{t}^{B}, Q_{t}\right)=$ $P_{t, k}$, and by negation, $\tau_{k}^{A}\left(q_{t}^{A}, Q_{t}\right)+\theta_{t}^{B} \tau_{k}^{B}\left(q_{t}^{B}, Q_{t}\right)<P_{t, k}$ implies $Q_{t, k}^{A}=0$. This implies that for all $\mathbf{Q}^{A} \geq 0$ :

$$
U_{Q_{t}}^{A}\left(Q^{A}-Q_{t}^{A}\right) \leq \frac{\lambda_{t}^{A}}{\frac{\partial W_{t}^{A}}{\partial U^{A}}}\left(P_{t}-\theta_{t}^{B} \tau_{t}^{B}\right)^{\prime}\left(Q^{A}-Q_{t}^{A}\right)
$$

Now, assume towards a contradiction that there exist an allocation $\left(q^{A}, Q^{A}\right)$ such that

$$
p_{t}^{\prime} q^{A}+\left(P_{t}-\theta_{t}^{B} \tau_{t}^{B}\right)^{\prime} Q^{A}+\theta_{t}^{A} \tau_{t}^{A \prime} Q_{t}^{B} \leq Y_{t}^{A}
$$

and,

$$
U^{A}\left(q^{A}, Q^{A}+Q_{t}^{B}\right)>U^{A}\left(q_{t}^{A}, Q_{t}^{A}+Q_{t}^{B}\right) .
$$

Then, by concavity of $U^{A}$, we have that:

$$
\begin{aligned}
U^{A}\left(q^{A}, Q^{A}+Q_{t}^{B}\right)-U^{A}\left(q_{t}^{A}, Q_{t}^{A}+Q_{t}^{B}\right) & \leq U_{q_{t}^{A}}^{A}\left(q^{A}-q_{t}^{A}\right)+U_{Q_{t}}^{A}\left(Q^{A}-Q_{t}^{A}\right) \\
& =\frac{\lambda_{t}^{A}}{\frac{\partial W_{t}^{A}}{\partial U_{t}^{A}}}\left[p_{t}^{\prime}\left(q^{A}-q_{t}^{A}\right)+\left(P_{t}-\theta_{t}^{B} \tau_{t}^{B}\right)^{\prime}\left(Q^{A}-Q_{t}^{A}\right)\right] \\
& \leq 0
\end{aligned}
$$

Again, we have a contradiction. 
Case (iii) For this last case, we define $\theta_{t}^{A}=\theta_{t}^{B}=1$, and,

$$
Y_{t}^{A}=p_{t}^{\prime} q_{t}^{A}+\left(P_{t}-\tau_{t}^{B}\right)^{\prime} Q_{t}^{A}+\tau_{t}^{A \prime} Q_{t}^{B}
$$

Assume, on the contrary, that there exist an allocation $\left(q^{A}, Q^{A}\right)$ such that

$$
p_{t}^{\prime} q^{A}+\left(P_{t}-\tau_{t}^{B}\right)^{\prime} Q^{A}+\tau_{t}^{A \prime} Q_{t}^{B} \leq Y_{t}^{A}
$$

and,

$$
U^{A}\left(q^{A}, Q^{A}+Q_{t}^{B}\right)>U^{A}\left(q_{t}^{A}, Q_{t}^{A}+Q_{t}^{B}\right) .
$$

Again, by concavity of $U^{A}$, we have that:

$$
\begin{aligned}
U^{A}\left(q^{A}, Q^{A}+Q_{t}^{B}\right)-U^{A}\left(q_{t}^{A}, Q_{t}^{A}+Q_{t}^{B}\right) & \leq U_{q_{t}^{A}}^{A}\left(q^{A}-q_{t}^{A}\right)+U_{Q_{t}}^{A}\left(Q^{A}-Q_{t}^{A}\right) \\
& =\frac{\lambda_{t}^{B}}{\frac{\partial W_{t}^{B}}{\partial U_{t}^{A}}}\left[p_{t}^{\prime}\left(q^{A}-q_{t}^{A}\right)+\left(P_{t}-\tau_{t}^{B}\right)^{\prime}\left(Q^{A}-Q_{t}^{A}\right)\right] \\
& \leq 0
\end{aligned}
$$

The equality follows from Lemma (1) and the fact that condition (6) must hold with equality for case (iii). This concludes the proof for individual A. The proof for individual $\mathrm{B}$ is analogous.

\section{A.2.2 Proof of statement 2}

Now assume that for each decision situation $t$ there exist indices $\theta_{t}^{A}, \theta_{t}^{B} \in[0,1]$ and incomes $Y_{t}^{A}, Y_{t}^{B}$ such that $\left\{q_{t}^{A}, q_{t}^{B}, Q_{t}^{A}, Q_{t}^{B}\right\}$ satisfies the definition of an equilibrium with transfers for utility functions $U^{A}, U^{B}$. We need to show that there exist caring functions $W_{t}^{A}$ and $W_{t}^{B}$ satisfying Assumption SC and consumption bundles $q_{t}^{A, A}, q_{t}^{A, B}, q_{t}^{B, B}, q_{t}^{B, A}$ (with $q_{t}^{A}=q_{t}^{A, A}+q_{t}^{A, B}$ and $\left.q_{t}^{B}=q_{t}^{B, A}+q_{t}^{B, B}\right)$ such that $\left\{q_{t}^{A, A}, q_{t}^{A, B}, q_{t}^{B, B}, q_{t}^{B, A}, Q_{t}^{A}, Q_{t}^{B}\right\}$ is a group equilibrium with caring.

We define the caring functions $W_{t}^{A}\left(U^{A}, U^{B}\right)=U^{A}+\theta_{t}^{B}\left(\mu_{t}^{A} / \mu_{t}^{B}\right) U^{B}$ and $W_{t}^{B}\left(U^{B}, U^{A}\right)=$ $U^{B}+\theta_{t}^{A}\left(\mu_{t}^{B} / \mu_{t}^{A}\right) U^{A}$. In this construction, $\mu_{t}^{A}$ and $\mu_{t}^{B}$ represent the marginal utilities of the numeraire for members $A$ and $B$ at equilibrium (i.e. $\frac{\partial U^{A}}{\partial q_{1}^{A}}=\mu_{t}^{A}$ and $\frac{\partial U^{B}}{\partial q_{1}^{B}}=\mu_{t}^{B}$ ). It is easy to see that these specifications satisfy Assumption SC as long as $\theta_{t}^{A}$ and $\theta_{t}^{B}$ are contained in the unit interval. Further, we choose $q_{t}^{A, A}=q_{t}^{A}, q_{t}^{B, B}=q_{t}^{B}, q_{t}^{A, B}=0$ and $q_{t}^{B, A}=0$. Let us focus on member A and assume on the contrary that there exist bundles $q^{A, A}, q^{A, B}, Q^{A}$ such that

$$
p_{t}^{\prime}\left(q^{A, A}+q^{A, B}+q_{t}^{B, B}\right)+P_{t}^{\prime}\left(Q^{A}+Q_{t}^{B}\right) \leq Y_{t}
$$

and,

$$
\begin{gathered}
U^{A}\left(q^{A, A}+q_{t}^{B, A}, Q^{A}+Q_{t}^{B}\right)+\theta_{t}^{B}\left(\mu_{t}^{A} / \mu_{t}^{B}\right) U^{B}\left(q_{t}^{B, B}+q^{A, B}, Q^{A}+Q_{t}^{B}\right)> \\
U^{A}\left(q_{t}^{A}, Q_{t}\right)+\theta_{t}^{B}\left(\mu_{t}^{A} / \mu_{t}^{B}\right) U^{B}\left(q_{t}^{B}, Q_{t}\right) .
\end{gathered}
$$


This gives,

$$
\begin{aligned}
& U^{A}\left(q^{A, A}, Q^{A}+Q_{t}^{B}\right)+\theta_{t}^{B}\left(\mu_{t}^{A} / \mu_{t}^{B}\right) U^{B}\left(q_{t}^{B}+q^{A, B}, Q^{A}+Q_{t}^{B}\right) \\
& -U^{A}\left(q_{t}^{A}, Q_{t}\right)-\theta_{t}^{B}\left(\mu_{t}^{A} / \mu_{t}^{B}\right) U^{B}\left(q_{t}^{B}, Q_{t}\right) \\
\leq & U_{q_{t}^{A}}^{A \prime}\left(q^{A, A}-q_{t}^{A}\right)+U_{Q_{t}}^{A \prime}\left(Q^{A}-Q_{t}^{A}\right)+\theta_{t}^{B}\left(\mu_{t}^{A} / \mu_{t}^{B}\right)\left[U_{q_{t}^{B}}^{B \prime} q^{A, B}+U_{Q_{t}}^{B \prime}\left(Q^{A}-Q_{t}^{A}\right)\right] \\
= & \mu_{t}^{A}\left[p_{t}^{\prime}\left(q^{A, A}-q_{t}^{A}\right)+\theta_{t}^{B} p_{t}^{\prime} q^{A, B}+\left(\tau^{A \prime}\left(q_{t}^{A}, Q_{t}\right)+\theta_{t}^{B} \tau^{B \prime}\left(q_{t}^{B}, Q_{t}\right)\right)\left(Q^{A}-Q_{t}^{A}\right)\right] \\
\leq & \mu_{t}^{A}\left[p_{t}^{\prime}\left(q^{A, A}+q^{A, B}-q_{t}^{A}\right)+P_{t}^{\prime}\left(Q^{A}-Q_{t}^{A}\right)\right] \\
\leq & 0 .
\end{aligned}
$$

The first inequality follows from concavity of the functions $U^{A}$ and $U^{B}$. The first equality follows from the first order conditions of programs DOP-A and DOP-B for the private goods. The second inequality follows from the fact that $\theta_{t}^{B} \leq 1$, the first order conditions of DOP-A for the public goods and the fact that $\tau_{t, k}^{A}+\theta_{t}^{B} \tau_{t, k}^{B}<P_{t, k}$ only if $Q_{t, k}^{A}=0$.

\section{A.3 Proof of Proposition 3}

$1 \Rightarrow 2$. The data set $S=\left\{p_{t}, P_{t}, q_{t}, Q_{t}\right\}_{t \in T}$ is rationalizable with caring. Because of Proposition 2, we have for any decision situation $t$ that the household allocation solves DOP-A and DOP-B. As before, let $U_{q_{t}^{M}}^{M}$ and $U_{Q_{t}}^{M}(M=A, B)$ be the subgradients for the function $U^{M}$ at bundle $\left(q_{t}^{M}, Q_{t}\right)$, and $\lambda_{t}^{A}$ and $\lambda_{t}^{B}$ the Lagrange multipliers for the budget constraints. We get as first order conditions, for each private good $j$ and public good $k$,

$$
\begin{aligned}
U_{q_{t, j}^{A}}^{A} & \leq \lambda_{t}^{A} p_{t, j}, \\
U_{q_{t, j}^{B}}^{B} & \leq \lambda_{t}^{B} p_{t, j}, \\
U_{Q_{t, k}}^{A} & \leq \lambda_{t}^{A}\left(P_{t, k}-\theta_{t}^{B} \tau^{B}\left(q_{t}^{B}, Q_{t}\right)\right), \\
U_{Q_{t, k}}^{B} & \leq \lambda_{t}^{B}\left(P_{t, k}-\theta_{t}^{A} \tau^{A}\left(q_{t}^{A}, Q_{t}\right)\right) .
\end{aligned}
$$

The inequalities are replaced by equalities in case the quantities of the goods under consideration are strictly positive. Next, concavity of the utility functions $U^{A}$ and $U^{B}$ implies, for all decision situations $t, v$

$$
\begin{gathered}
U^{A}\left(q_{t}^{A}, Q_{t}\right)-U^{A}\left(q_{v}^{A}, Q_{v}\right) \leq U_{q_{v}^{A}}^{A \prime}\left(q_{t}^{A}-q_{v}^{A}\right)+U_{Q_{v}}^{A \prime}\left(Q_{t}-Q_{v}\right), \\
U^{B}\left(q_{t}^{B}, Q_{t}\right)-U^{B}\left(q_{v}^{B}, Q_{v}\right) \leq U_{q_{v}^{B}}^{B \prime}\left(q_{t}^{B}-q_{v}^{B}\right)+U_{Q_{v}}^{B \prime}\left(Q_{t}-Q_{v}\right) .
\end{gathered}
$$

For all $t$, define $U_{Q_{t}}^{A} / \lambda_{t}^{A}=\tau_{t}^{A}$ and $U_{Q_{t}}^{B} / \lambda_{t}^{B}=\tau_{t}^{B}, U^{A}\left(q_{t}^{A}, Q_{t}\right)=U_{t}^{A}$ and $U^{B}\left(q_{t}^{B}, Q_{t}\right)=U_{t}^{B}$. This gives,

$$
\begin{aligned}
& U_{t}^{A}-U_{v}^{A} \leq \lambda_{v}^{A}\left(p_{v}^{\prime}\left(q_{t}^{A}-q_{v}^{A}\right)+\tau_{t}^{A \prime}\left(Q_{t}-Q_{v}\right)\right), \\
& U_{t}^{B}-U_{v}^{B} \leq \lambda_{v}^{B}\left(p_{v}^{\prime}\left(q_{t}^{B}-q_{v}^{B}+\tau_{t}^{B}\left(Q_{t}-Q_{v}\right)\right) .\right.
\end{aligned}
$$

To see that this obtains S.3, we make use of the Afriat Theorem (see Afriat (1967) and Varian (1982)). Specifically, the inequalities in (15)-(16) are so-called Afriat inequalities, 
and the Afriat Theorem implies that these inequalities are satisfied for all $t, v$ if and only if the sets $\left\{p_{t}, \tau_{t}^{A}, q_{t}^{A}, Q_{t}\right\}_{t \in T}$ and $\left\{p_{t}, \tau_{t}^{B}, q_{t}^{B}, Q_{t}\right\}_{t \in T}$ satisfy GARP.

Moreover, at the equilibrium, if $\tau_{t, k}^{A}+\theta_{t}^{B} \tau_{t, k}^{B}<P_{t, k}$, then $Q_{t, k}^{A}=0$ and, thus, $Q_{t, k}^{B}=$ $Q_{t, k}>0$. Then, the first order condition for $k$ in DOP-B must be binding, so that $\theta_{t}^{A} \tau_{t, k}^{A}+\tau_{t, k}^{B}=P_{t, k}$. This obtains the first part of S.2. Reversing the roles of $A$ and $B$ shows the other part of S.2. Similarly, one can verify S.4 and S.5.

$2 \Rightarrow 1$. Because the GARP conditions in (S.3) are satisfied, the Afriat Theorem (mentioned above) tells us that there exist positive numbers $U_{t}^{A}, U_{t}^{B}$ and strictly positive numbers $\lambda_{t}^{A}$ and $\lambda_{t}^{B}$ such that the following Afriat inequalities hold:

$$
\begin{aligned}
& U_{t}^{A}-U_{v}^{A} \leq \lambda_{v}^{A}\left(p_{v}^{\prime}\left(q_{t}^{A}-q_{v}^{A}\right)+\tau_{v}^{A \prime}\left(Q_{t}-Q_{v}\right)\right), \\
& U_{t}^{B}-U_{v}^{B} \leq \lambda_{v}^{B}\left(p_{v}^{\prime}\left(q_{t}^{B}-q_{v}^{B}\right)+\tau_{v}^{B \prime}\left(Q_{t}-Q_{v}\right)\right) .
\end{aligned}
$$

Then, define the functions $U^{A}$ and $U^{B}$ such that:

$$
\begin{aligned}
& U^{A}\left(q^{A}, Q\right)=\min _{v \in T}\left\{U_{v}^{A}+\lambda_{v}^{A}\left(p_{v}^{\prime}\left(q^{A}-q_{v}^{A}\right)+\tau_{v}^{A \prime}\left(Q-Q_{v}\right)\right)\right\}, \\
& U^{B}\left(q^{B}, Q\right)=\min _{v \in T}\left\{U_{v}^{B}+\lambda_{v}^{B}\left(p_{v}^{\prime}\left(q^{B}-q_{v}^{B}\right)+\tau_{v}^{B \prime}\left(Q-Q_{v}\right)\right)\right\} .
\end{aligned}
$$

Notice that $U^{A}$ and $U^{B}$ are continuous, concave, strictly monotone and that for all $t \in T$, $U^{A}\left(q_{t}^{A}, Q_{t}\right)=U_{t}^{A}$ and $U^{B}\left(q_{t}^{B}, Q_{t}\right)=U_{t}^{B}$. See, for example, Varian (1982).

We need to show that the functions $U^{A}$ and $U^{B}$ provide a rationalization of the data set. For brevity, we only provide the argument for $U^{A}$, but a straightforwardly analogous reasoning applies to $U^{B}$. For all $t \in T$, define $Q_{t}^{A}$ and $Q_{t}^{B}$ so that if $\tau_{t, k}^{A}+\theta_{t}^{B} \tau_{t, k}^{B}<P_{t, k}$ then $Q_{t, k}^{A}=0$ and $Q_{t, k}^{B}=Q_{t, k}$, and if $\theta_{t}^{A} \tau_{t, k}^{A}+\tau_{t, k}^{B}<P_{t, k}$ then $Q_{t, k}^{B}=0$ and $Q_{t, k}^{A}=Q_{t, k}$ (see S.4 and S.5). (If $\tau_{t, k}^{A}+\theta_{t}^{B} \tau_{t, k}^{B}=P_{t}$ and $\theta_{t}^{A} \tau_{t, k}^{A}+\tau_{t, k}^{B}=P_{t, k}$ then we can randomly allocate $Q_{t, k}$ between $Q_{t, k}^{A}$ and $Q_{t, k}^{B}$.) Next, consider $t \in T$ and a bundle $\left(q^{A}, Q^{A}\right)$ with $Q=Q^{A}+Q_{t}^{B}$ such that

$p_{t}^{\prime} q^{A}+\sum_{k}\left[\left(P_{t, k}-\theta_{t}^{B} \tau_{t, k}^{B}\right) Q_{k}^{A}+\theta_{t}^{A} \tau_{t, k}^{A} Q_{t, k}^{B}\right) \leq p_{t}^{\prime} q_{t}^{A}+\sum_{k}\left[\left(P_{t, k}-\theta_{t}^{B} \tau_{t, k}^{B}\right) Q_{t, k}^{A}+\theta_{t}^{A} \tau_{t, k}^{A} Q_{t, k}^{B}\right)$,

or equivalently,

$$
p_{t}^{\prime} q^{A}+\sum_{k}\left[\left(P_{t, k}-\theta_{t}^{B} \tau_{t, k}^{B}\right) Q_{k}^{A}\right) \leq p_{t}^{\prime} q_{t}^{A}+\sum_{k}\left[\left(P_{t, k}-\theta_{t}^{B} \tau_{t, k}^{B}\right) Q_{t, k}^{A}\right) .
$$

Then, we have to prove that $U^{A}\left(q^{A}, Q\right) \leq U^{A}\left(q_{t}^{A}, Q_{t}\right)$. To obtain this result, we first note that, by construction, $\tau_{t}^{A} Q_{t}^{A}=\left(P_{t}-\theta_{t}^{B} \tau_{t}^{B}\right) Q_{t}^{A}$. Thus, because $\tau_{t, k}^{A}+\theta_{t}^{B} \tau_{t, k}^{B} \leq P_{t, k}$ (which implies $\left.\tau_{t}^{A} Q^{A} \leq\left(P_{t}-\theta_{t}^{B} \tau_{t}^{B}\right) Q^{A}\right)$, we get $\tau_{t}^{A}\left(Q^{A}-Q_{t}^{A}\right) \leq\left(P_{t}-\theta_{t}^{B} \tau_{t}^{B}\right)^{\prime}\left(Q^{A}-Q_{t}^{A}\right)$. Using this, we obtain

$$
\begin{aligned}
U^{A}\left(q^{A}, Q\right) & =\min _{v \in T}\left\{U_{v}^{A}+\lambda_{v}^{A}\left(p_{v}^{\prime}\left(q^{A}-q_{v}^{A}\right)+\tau_{v}^{A \prime}\left(Q-Q_{v}\right)\right)\right\} \\
& \leq U_{t}^{A}+\lambda_{t}^{A}\left(p_{t}^{\prime}\left(q^{A}-q_{t}^{A}\right)+\tau_{t}^{A \prime}\left(Q-Q_{t}\right)\right) \\
& =U_{t}^{A}+\lambda_{t}^{A}\left(p_{t}^{\prime}\left(q^{A}-q_{t}^{A}\right)+\tau_{t}^{A \prime}\left(Q^{A}-Q_{t}^{A}\right)\right) \\
& \leq U_{t}^{A}+\lambda_{t}^{A}\left(p_{t}^{\prime}\left(q^{A}-q_{t}^{A}\right)+\left(P_{t}-\theta_{t}^{B} \tau_{t}^{B}\right)^{\prime}\left(Q^{A}-Q_{t}^{A}\right)\right) \\
& \leq U_{t}^{A} .
\end{aligned}
$$

This provides the wanted result, i.e. $\left\{q_{t}^{A}, Q_{t}^{A}\right\}$ solves DOP-A. 


\section{B Mixed integer programming characterization}

In this appendix, we reformulate the conditions in Proposition 3 in mixed integer programming (MIP) terms. To obtain this MIP formulation, we define the binary variables $x_{t, v}^{M} \in\{0,1\}$, with $x_{t, v}^{M}=1$ interpreted as $\left(q_{t}^{M}, Q_{t}\right) R^{M}\left(q_{v}^{M}, Q_{v}\right)$ where $R^{M}$ is the revealed preference relation for individual $M \in\{A, B\}$. Then, a data set $S$ satisfies the necessary and sufficient condition for rationalizability as given by Proposition 3 if and only if the following MIP problem is feasible:

For all decision situations $t, v$ and public goods $k$ there exist strictly positive vectors $\tau_{t}^{A} \tau_{t}^{B} \in$ $\mathbb{R}_{++}^{K}$, binary variables $b_{t, k}, x_{t, v}^{A}, x_{t, v}^{B} \in\{0,1\}$, and parameters $\theta_{t}^{A}, \theta_{t}^{B} \in[0,1]$ such that: ${ }^{23}$

$$
\begin{aligned}
& \tau_{t}^{A}+\theta_{t}^{B} \tau_{t}^{B} \leq P_{t}, \\
& \theta_{t}^{A} \tau_{t}^{A}+\tau_{t}^{B} \leq P_{t}, \\
& P_{t, k}-\tau_{t, k}^{A}-\theta_{t}^{B} \tau_{t, k}^{B} \leq b_{t, k} C_{t}, \\
& P_{t, k}-\theta_{t}^{A} \tau_{t, k}^{A}-\tau_{t, k}^{B} \leq\left(1-b_{t, k}\right) C_{t}, \\
& q_{t}^{A}+q_{t}^{B}=q_{t}, \\
& p_{t}^{\prime}\left(q_{t}^{M}-q_{v}^{M}\right)+\tau_{t}^{M \prime}\left(Q_{t}-Q_{v}\right)<x_{t, v}^{M} C_{t}, \\
& x_{t, s}^{M}+x_{s, v}^{M} \leq 1+x_{t, v}^{M}, \\
& \left(1-x_{t, v}^{M}\right) C_{v} \geq p_{v}^{\prime}\left(q_{v}^{M}-q_{t}^{M}\right)+\tau_{v}^{M \prime}\left(Q_{v}-Q_{t}\right),
\end{aligned}
$$

with $C_{t}$ a given number for which $C_{t}>P_{t, k}$ and $C_{t}>Y_{t}$ for all $t, k$.

The explanation is as follows. Constraint (21) imposes that the private consumption bundles $q_{t}^{A}$ and $q_{t}^{B}$ sum to the observed aggregate quantities $q_{t}$, as required by condition S.1. Further, constraints (17)-(20) comply with condition S.2 in Proposition 3. Specifically, (17) and (18) impose the given upper bound restriction for $\tau_{t}^{A}$ and $\tau_{t}^{B}$. Next, (19) imposes $P_{t, k} \leq \tau_{t, k}^{A}+\theta_{t}^{B} \tau_{t, k}^{B}$ if $z_{t, k}=0$, while (20) imposes $P_{t, k} \leq \theta_{t}^{A} \tau_{t, k}^{A}+\tau_{t, k}^{B}$ if $z_{t, k}=1$. Because $z_{t, k} \in\{0,1\}$, this implies $\max \left\{\tau_{t, k}^{A}+\theta_{t}^{B} \tau_{t, k}^{B} ; \tau_{t, k}^{B}+\theta_{t}^{A} \tau_{t, k}^{A}\right\}=P_{t, k}$ and thus condition S.2 is satisfied. Finally, constraints (22)-(24) correspond to the GARP conditions for each individual $M(=A$ or $B)$ (condition S.3 in Proposition 3). Specifically, (22) states that $p_{t}^{\prime}\left(q_{t}^{M}-q_{v}^{M}\right)+\tau_{t}^{M \prime}\left(Q_{t}-Q_{v}\right) \geq 0$ implies $x_{t, v}^{M}=1$ (or $\left.\left(q_{t}^{M}, Q_{t}\right) R^{M}\left(q_{v}^{M}, Q_{v}\right)\right)$. Next, constraint (23) imposes transitivity of the individual revealed preference relations $R^{M}$ : if $x_{t, s}^{M}=1$ (i.e. $\left.\left(q_{t}^{M}, Q_{t}\right) R^{M}\left(q_{s}^{M}, Q_{s}\right)\right)$ and $x_{s, v}^{M}=1$ (i.e. $\left.\left(q_{s}^{M}, Q_{s}\right) R^{M}\left(q_{v}^{M}, Q_{v}\right)\right)$ then $x_{t, v}^{M}=1$ (i.e. $\left.\left(q_{t}^{M}, Q_{t}\right) R^{M}\left(q_{v}^{M}, Q_{v}\right)\right)$. And (24) requires $p_{v}^{\prime}\left(q_{v}^{M}-q_{t}^{M}\right)+\tau_{v}^{M \prime}\left(Q_{v}-Q_{t}\right) \leq 0$ if $x_{t, v}^{M}=1$ (i.e. $\left.\left(q_{t}^{M}, Q_{t}\right) R^{M}\left(q_{v}^{M}, Q_{v}\right)\right)$.

Clearly, all constraints are linear for fixed $\theta_{t}^{A}$ and $\theta_{t}^{B}$. Linearity implies that the above program can be solved by standard MIP methods for a given data set $S$. See also our discussion in the main text on conducting a grid search for $\theta_{t}^{A}, \theta_{t}^{B} \in[0,1]$.

\footnotetext{
${ }^{23}$ The strict inequality $p_{t}^{\prime}\left(q_{t}^{M}-q_{v}^{M}\right)+\tau t^{M}\left(Q_{t}-Q_{v}\right)<x_{t, v}^{M} C_{t}$ is difficult to use in IP analysis. Therefore, in practice we can replace it with $p_{t}^{\prime}\left(q_{t}^{M}-q_{v}^{M}\right)+\tau_{t}^{M \prime}\left(Q_{t}-Q_{v}\right)+\epsilon \leq x_{t, v}^{M} C_{t}$ for $\epsilon(>0)$ arbitrarily small.
} 


\section{Computing the goodness-of-fit index $f^{*}$}

For our experiment, let $S=\left\{p_{t}, P_{t}, q_{t}^{M}, Q_{t}\right\}_{t \in T, M \in\{A, B\}}$ represent one data set from the joint choice experiment and let $S^{M}=\left\{p_{s}, P_{s}, q_{s}^{M}, Q_{s}\right\}_{s \in T^{M}}$ represent one data set from $M$ 's individual choice experiment. We write $S^{\text {pool }}=\left\{S, S^{A}, S^{B}\right\}$. Our program will compute the lowest value of $f$ (see our discussion of Definition 5) such that the data in $S$ (i.e. (25)-(32) below) as well as all pairwise comparisons of joint bundles $t \in T$ and individual bundles $s \in T^{A}$ and $u \in T^{B}$ (i.e. (33)-(40)) are consistent with our testable implications. Using the integer programming formulation of Appendix B, we obtain the test by defining the binary variables $x_{t, s}^{M} \in\{0,1\}$, with $x_{t, s}^{M}=1$ interpreted as $\left(q_{t}^{M}, Q_{t}\right) \widetilde{R^{M}}\left(q_{s}^{M}, Q_{s}\right)$ where $\widetilde{R^{M}}$ is the revealed preference relation for individual $M \in\{A, B\}$ (see Definition 5):

For all joint decision situations $t, v, w \in T$, individual decision situations $s \in T^{A}, u \in T^{B}$ and public goods $k$, we look for

- strictly positive vectors $\tau_{t}^{A}, \tau_{t}^{B} \in \mathbb{R}_{++}^{K}$,

- binary variables $b_{t, k}, x_{t, v}^{A}, x_{t, v}^{B}, x_{t, s}^{A}, x_{t, u}^{B}, x_{s, t}^{A}, x_{u, t}^{B} \in\{0,1\}$,

- parameters $\theta \in[0,1]$,

- non-negative numbers $c_{t}^{A}, c_{t}^{B}, c_{s}^{A}, c_{u}^{B}$,

that solve

$$
f^{*}=\min \sum_{t \in T} \sum_{M \in A, B}\left(c_{t}^{M}\right)+\sum_{s \in T^{A}} c_{s}^{A}+\sum_{u \in T^{B}} c_{u}^{B}
$$

subject to

$$
\begin{aligned}
& \tau_{t}^{A}+\theta \tau_{t}^{B} \leq P_{t}, \\
& \theta \tau_{t}^{A}+\tau_{t}^{B} \leq P_{t}, \\
& P_{t, k}-\tau_{t, k}^{A}-\theta \tau_{t, k}^{B} \leq b_{t, k} C_{t}, \\
& P_{t, k}-\theta \tau_{t, k}^{A}-\tau_{t, k}^{B} \leq\left(1-b_{t, k}\right) C_{t}, \\
& q_{t}^{A}+q_{t}^{B}=q_{t},
\end{aligned}
$$

and, for each individual $M=A, B$,

$$
\begin{aligned}
& p_{t}^{\prime}\left(q_{t}^{M}-q_{v}^{M}\right)+\tau_{t}^{M \prime}\left(Q_{t}-Q_{v}\right)-c_{t}^{M}<x_{t, v}^{M} C_{t}, \\
& x_{t, w}^{M}+x_{w, v}^{M} \leq 1+x_{t, v}^{M}, \\
& \left(1-x_{t, v}^{M}\right) C_{v} \geq p_{v}^{\prime}\left(q_{v}^{M}-q_{t}^{M}\right)+\tau_{v}^{M \prime}\left(Q_{v}-Q_{t}\right)-c_{v}^{M}
\end{aligned}
$$

and, for individual $A$,

$$
\begin{aligned}
& p_{t}^{\prime}\left(q_{t}^{A}-q_{s}^{A}\right)+\tau_{t}^{A \prime}\left(Q_{t}-Q_{s}\right)-c_{t}^{A}<x_{t, s}^{A} C_{t}, \\
& \left(1-x_{t, s}^{A}\right) C_{s} \geq p_{s}^{\prime}\left(q_{s}^{A}-q_{t}^{A}\right)+P_{s}^{\prime}\left(Q_{s}-Q_{t}\right)-c_{s}^{A}, \\
& p_{s}^{\prime}\left(q_{s}^{A}-q_{t}^{A}\right)+P_{s}^{\prime}\left(Q_{s}-Q_{t}\right)-c_{s}^{A}<x_{s, t}^{A} C_{s}, \\
& \left(1-x_{s, t}^{A}\right) C_{t} \geq p_{t}^{\prime}\left(q_{t}^{A}-q_{s}^{A}\right)+\tau_{t}^{A \prime}\left(Q_{t}-Q_{s}\right)-c_{t}^{A},
\end{aligned}
$$


and, for individual $B$,

$$
\begin{aligned}
& p_{t}^{\prime}\left(q_{t}^{B}-q_{u}^{B}\right)+\tau_{t}^{B \prime}\left(Q_{t}-Q_{u}\right)-c_{t}^{B}<x_{t, u}^{B} C_{t}, \\
& \left(1-x_{t, u}^{B}\right) C_{u} \geq p_{u}^{\prime}\left(q_{u}^{B}-q_{t}^{B}\right)+P_{u}^{\prime}\left(Q_{u}-Q_{t}\right)-c_{u}^{B}, \\
& p_{u}^{\prime}\left(q_{u}^{B}-q_{t}^{B}\right)+P_{u}^{\prime}\left(Q_{u}-Q_{t}\right)-c_{u}^{B}<x_{u, t}^{B} C_{u}, \\
& \left(1-x_{u, t}^{B}\right) C_{t} \geq p_{t}^{\prime}\left(q_{t}^{B}-q_{u}^{B}\right)+\tau_{t}^{B \prime}\left(Q_{t}-Q_{u}\right)-c_{t}^{B},
\end{aligned}
$$

with $C_{t}$ a given number for which $C_{t}>P_{t, k}$ and $C_{t}>Y_{t}$ for all $t, k$.

Conditions (25)-(32) are direct translations of conditions (17)-(24), but now taking into account the goodness-of-fit specification in Definition 5 and implementing the restriction $\theta^{A}=\theta^{B}=\theta$ (which we use in our experimental application). Conditions (33)-(40) impose revealed preference consistency conditions between the joint and individual choices. In particular, they imposes WARP (Weak Axiom of Revealed Preference) restrictions across the two choice sets: if a choice from the single choice environment is revealed preferred over a choice from the joint decision environment, then this joint choice is not also revealed preferred over the individual choice (and vice versa). ${ }^{24}$

This optimization problem is solved with $\theta=1$ to compute $f_{\text {coop }}^{*}$, with $\theta=0$ to compute $f_{\text {noncoop }}^{*}$, and by conducting a grid search for $\theta \in[0,1]$ to compute $f_{\text {caring }}^{*}$. In our empirical application, we obtain identification of $\left[\theta^{l b}, \theta^{u b}\right]$ by taking the values of $\theta$ from this grid that yield the smallest objective function value (i.e. the best empirical fit).

\footnotetext{
${ }^{24}$ Generally, WARP is a weaker rationalizability requirement than GARP (which we use in Proposition 3). In theory, imposing GARP on pairwise comparisons between the individual and joint decision environment could further narrow the bounds on $\theta$. In practice, however, this severely increases the number of constraints and, hence, the computational burden associated with our rationalizability conditions. Moreover, for the choice data of our empirical application, using GARP instead of WARP leaves our main conclusions virtually unchanged.
} 


\section{References}

[1] Adams, A. (2015), Mutually consistent revealed preference bounds, IFS working paper W15/20.

[2] Afriat, S. (1967), The Construction of Utility Functions from Expenditure Data, International Economic Review 8, 67-77.

[3] Afriat, S. (1973), On a System of Inequalities in Demand Analysis: An Extension of the Classical Method, International Economic Review 14, 460-472.

[4] Angerer, S., D. Glätzle-Rützler, P. Lergetporer and M. Sutter (2016), Cooperation and discrimination within and across language borders: Evidence from children in a bilingual city, European Economic Review 90, 254-264.

[5] Apps, P. and R. Rees (1988), Taxation and the Household, Journal of Public Economics 35, 355-369.

[6] Apps, P. and R. Rees (2009), Public economics and the household, Cambridge University Press.

[7] Becker, G. (1981), Altruism in the family and selfishness in the market, Economica 48, 1-15.

[8] Bergstrom, T. (1976), Theory and measurement of economic externalities, Academic Press.

[9] Bergstrom, T., L. Blume and H. Varian (1986), On the private provision of public goods, Journal of Public Economics 29, 25-49.

[10] Blundell, R., M. Browning and I. Crawford (2003), Nonparametric Engel Curves and. Revealed Preference, Econometrica 71, 205-240.

[11] Blundell, R., M. Browning and I. Crawford (2008), Best nonparametric bounds on demand responses, Econometrica 76, 1227-1262.

[12] Blundell, R., P.-A. Chiappori and C. Meghir (2005), Collective labor supply with children, Journal of Political Economy 113, 1277-1306.

[13] Boone, J., K. van der Wiel, A. van Soest and F. Vermeulen (2014), Kinky choices, dictators and split might. A noncooperative model for household consumption and labor supply, IZA journal of Labor Economics 3, 1-19.

[14] Bourguignon, F. (1984), Rationalité individuelle our rationalité stratégique: le cas de l'offre familiale de travail, Revue Economique 35, 147-162.

[15] Browning, M. (2000), The saving behaviour of a two-person household, Scandinavian Journal of Economics 102, 235-251. 
[16] Browning, M, F. Bourguignon, P.-A. Chiappori and V. Lechene (1994), A structural model of intrahousehold allocation, Journal of Political Economy 102, 1067-1096.

[17] Browning, M. and P.-A. Chiappori (1998), Efficient Intra-houshold Allocations: A General Characterisation and Empirical Tests, Econometrica 66, 1241-1278.

[18] Browning, M, P.-A. Chiappori and V. Lechene (2006), Collective and Unitary Models: A Clarification, Review of Economics of the Household 4, 5-14.

[19] Browning, M, P.-A. Chiappori and V. Lechene (2010), Distributional effects in household models: separate spheres and income pooling, Economic Journal 120, 786-799.

[20] Browning, M. and V. Lechene (2001), Caring and sharing: tests between alternative models of intrahousehold allocation, University of Oxford discussion paper 70, Department of Economics.

[21] Bruyneel, S., Cherchye, L., Cosaert, S. De Rock, B. and S. Dewitte (2017), Measuring the willingness-to-pay for others' consumption: An application to joint decisions of children, Quantitative Economics forthcoming.

[22] Calvert, S. L. (2008), Children as consumers: Advertising and marketing, Future of Children 18, 205-234.

[23] Chen, Z. and F. Woolley (2001), A Cournot-Nash equilibrium of family decision making, Economic Journal 111, 722-748.

[24] Cherchye, L., T. Demuynck and B. De Rock (2011), Revealed preference analysis of noncooperative household consumption, Economic Journal 121, 1073-1096.

[25] Cherchye, L., T. Demuynck, B. De Rock and F. Vermeulen (2017), Household consumption when the marriage is stable, American Economic Review 107, 1507-1534.

[26] Cherchye, L., B. De Rock, A. Lewbel and F. Vermeulen (2015), Sharing rule identification for general collective consumption models, Econometrica 83, 2001-2041.

[27] Cherchye, L., B. De Rock and F. Vermeulen (2007), The collective model of household consumption: a nonparametric characterization, Econometrica 75, 553-574.

[28] Cherchye, L., B. De Rock, and F. Vermeulen (2009), Opening the black box of intrahousehold decision-making: theory and non-parametric empirical tests of general collective consumption models, Journal of Political Economy 117, 1074-1104.

[29] Cherchye, L., B. De Rock and F. Vermeulen (2011), The revealed preference approach to collective consumption behavior: testing and sharing rule recovery, Review of Economic Studies 78, 176-198.

[30] Chiappori, P.-A. (1988), Rational household labor supply, Econometrica 56, 63-89.

[31] Chiappori, P.-A. (1992), Collective labor supply and welfare, Journal of Political Economy 100, 437-467. 
[32] Chiappori, P.-A. and I. Ekeland (2006), The micro economics of group behavior: general characterization, Journal of Economic Theory 130, 1-26.

[33] Chiappori, P.-A. and I. Ekeland (2009), The micro economics of efficient group behavior: identification, Econometrica 77, 763-799.

[34] Choi, S., S. Kariv, W. Muller, and D. Silverman (2014), Who Is (More) Rational?, American Economic Review 104, 1518-1550.

[35] Czermak, S., F. Feri, D. Glätzle-Rützler and M. Sutter (2016), How strategic are children and adolescents? Experimental evidence from normal-form games, Journal of Economic Behavior and Organization 128, 265-285.

[36] d'Aspremont, C. and R. Dos Santos Ferreira (2014), Household behavior and individual autonomy: an extended Lindahl mechanism, Economic Theory 55, 643-664.

[37] d'Aspremont, C. and R. Dos Santos Ferreira (2017), Enlarging the collective model of household behaviour: a revealed preference analysis, working paper.

[38] Diewert, W.E. (1973), Afriat and revealed preference theory, Review of Economic Studies 40, 419-426.

[39] Fehr, E., D. Glätzle-Rützler and M. Sutter (2013), The development of egalitarianism, altruism, spite and parochialism in childhood and adolescence, European Economic Review 64, 369-383.

[40] Flinn, C., P. Todd and W. Zhang (2017), Personality Traits, Intra-household Allocation and the Gender Wage Gap, working paper.

[41] Halevy, Y., D. Persitz and L. Zrill (2016), Parametric Recoverability of Preferences, Journal of Political Economy, forthcoming.

[42] Harbaugh, W. T., K. Krause and T. R. Berry (2001), GARP for kids: On the development of rational choice behavior, American Economic Review 91, 1539-1545.

[43] Houthakker, H. S. (1950), Revealed preference and the utility function, Economica $17,159-174$.

[44] Kemp, M. C. (1984), A note of the theory of international transfers, Economics Letters $14,259-262$.

[45] Kooreman, P. and A. Kapteyn (1990), On the empirical implementation of some game theoretic models of household labor supply, Journal of Human Resources 25, 584-598.

[46] Lechene, V. and I. Preston (2005), Household Nash Equilibrium with Voluntarily Contributed Public Goods, IFS working paper 05/06.

[47] Lechene, V. and I. Preston (2011), Non Cooperative Household Demand, Journal of Economic Theory 146, 504-527. 
[48] Leuthold, J (1968), An empirical study of formula transfers and the work decision of the poor, Journal of Human Resources 3, 312-323.

[49] Lundberg, S. and R. Pollak (1993), Separate Spheres Bargaining and the Marriage Market, Journal of Political Economy 101, 988-1010.

[50] Manser, M. and M. Brown (1980), Marriage and household decision making: a bargaining analysis, International Economic Review 21, 31-44.

[51] McElroy, M. B. and M. Horney (1981), Nash-bargained household decisions: towards a generalization of the theory of demand, International Economic Review 22, 333-349.

[52] Papke, L. E. and J. M. Wooldridge (1996), Econometric methods for fractional response variables with an application to $401(\mathrm{k})$ plan participation rates, Journal of Applied Econometrics 11, 619-632.

[53] Samuelson, P. A. (1938), A Note on the pure theory of consumer behavior, Economica $5,61-71$.

[54] Samuelson, P. A. (1954), The pure theory of public expenditures, Review of Economics and Statistics 36, 387-389.

[55] Seguin, J., L. Arseneault and R. Tremblay (2007), The contribution of "cool" and "hot" components of decision-making in adolescence: Implications for developmental psychopathology, Cognitive Development 20, 530-543.

[56] Ulph, D. (1988), A general non-cooperative Nash model of household consumption behavior, University of Bristol, Economics Decision Paper 88/205.

[57] Varian, H. (1982), The Nonparametric Approach to Demand Analysis, Econometrica 50, 945-973.

[58] Varian, H. (1990), Goodness-of-fit in Optimizing Models, Journal of Econometrics 46, 125-140.

[59] Wouters, E., J. Larsen, S. Kremers, P. Dagnelie and R. Geenen (2010), Peer influence on snacking behavior in adolescence, Appetite 55, 11-17. 
Copyright (c) 2017 @ the author(s). Discussion papers are in draft form. This discussion paper is distributed for purposes of comment and discussion only. It may not be reproduced without permission of the copyright holder. Copies of working papers are available from the author. 